\title{
ENTRAINMENT OF FREQUENCY IN SINGULARLY PERTURBED SYSTEMS
}

\author{
Jane Cronin
}

\begin{abstract}
Conditions are obtained to insure the existence of entrainment of frequency under the following constraints: (i) the difference between the forcing frequency and the natural frequency is not small; (ii) the forcing term is not small; (iii) the amplitude of the entrained oscillations is required to be almost the same as the amplitude of the natural frequency oscillations. Such entrainment of frequency occurs in electrically active cells; for example, the Purkinje fiber has a natural frequency of oscillation but is affected by periodic pacemaker impulses. It is shown that classes of singularly perturbed differential equations (Liénard equations), where the natural oscillation is a relaxation oscillation, satisfy the derived conditions.
\end{abstract}

\section{Introduction}

Entrainment of frequency has been observed in physical systems for centuries and has been studied mathematically for over a hundred years. The phenomenon can be described as follows: suppose a periodic force is impressed upon a physical system which has a natural frequency of oscillation and that the natural frequency is different from the frequency of the impressed force. If the system then oscillates with the frequency of the impressed force, we say that entrainment of frequency occurs. The classical mathematical study, which was initiated in the work of Poincaré [30], is concerned with the case in which the difference between the frequency of the forcing term and the natural frequency is small and the forcing term itself is small. A large number of studies of this kind, motivated by problems in sound, celestial mechanics, and electrical circuit theory, have been made. For descriptions of this work and summaries of earlier publications, see Roseau [31, 32], Malkin [23], Minorsky [24, 25], Leimanis and Minorsky [19], Coddington and Levinson [8], Hale [15, 16], and Cronin [11].

It has long been known (see van der Pol and van der Mark [35]) that entrainment of frequency may occur in which the difference between the frequency of the forcing term and the natural frequency is large, the forcing term is not small and yet the amplitude of the entrained oscillation will be almost the same as the amplitude of the natural frequency oscillation. Straightforward inspection shows that the classical mathematical study is not applicable in such cases. However, as we will see, entrainment of frequency of the kind described by van der Pol and van der Mark does occur in mathematical models which are singularly perturbed.

It is important to study problems of this kind because many mathematical models in mechanics, electrical circuits, and biology are singularly perturbed differential equations. In particular, quantitative studies of biological cells often yield mathematical models that are singularly perturbed systems. The original Hodgkin-Huxley equations

Received March 10, 1995, revised April 30, 1996.

1991 Mathematics Subject Classification: 34C15, 34E15.

Key words and phrases: entrainment of frequency, singularly perturbed equations, relaxation oscillation. 
(Hodgkin and Huxley [17]), which model a nerve cell, are singularly perturbed equations. Many other quantitative studies have been made in which mathematical models analogous to the Hodgkin-Huxley equations are derived. These include models of the myelinated nerve (Frankenhaeuser and Huxley [14]), the striated muscle fiber (Adrian and Peachey [2]), components of the mammalian heart (Noble [27, 28], McAllister et al. [22], Beeler and Reuter [3], Yanagihara et al. [36], DiFrancesco and Noble [12], Noble and Noble [29]), and neuronal networks (Traub and Miles [34]). The question of entrainment of frequency of the type described above arises in the study of such models. For example, such entrainment occurs in the cardiac Purkinje fiber whose frequency of oscillation is governed by pacemaker impulses.

The purpose of this paper is to investigate conditions under which entrainment of frequency of the kind described by van der Pol and van der Mark occurs and is stable. We will describe such conditions and then show that they are satisfied by a class of singularly perturbed equations. In Section 2, we use the Poincaré method to study locally the periodic perturbation of an autonomous system. That is, we assume that the $n$-vector equation

$$
\frac{d x}{d t}=f(x)
$$

has a solution $\bar{x}(t)$ of period $T$, and we investigate the equation

$$
\frac{d x}{d t}=f(x)+\mu g(t, \mu)
$$

where $\mu$ is a small positive parameter and $g(t, \mu)$ has period $T(\mu)$ where $T(\mu)$ is a differentiable function of $\mu$ with $T(0)=T$. We want to determine if, for sufficiently small $\mu$, the perturbed equation has a solution of period $T(\mu)$ and is near $\bar{x}(t)$. This problem is a special case of the general class of problems that can be studied by using the Poincaré method. For a complete development of the Poincaré method, see Roseau [31] and, for many applications, see Roseau [32]. The problem we study in Section 2 is a special case of the discussion in Roseau [31, Chapitre 18, especially pp. 247-250]. The method used here is particularly adapted for practical calculations which are needed in later sections of this paper.

It should be noted that the problem studied in Roseau [31, Chapitre 18] was studied earlier and in detail by J. Haag. For references to Haag's work, see [31, p. 251].

In Section 3, we study the continuation of the local problem considered in Section 2. That is, we allow the parameter $\mu$ to take all values in the interval $[0,1]$. To obtain the results in Sections 2 and 3, we introduce various hypotheses, and in Section 4 we discuss the significance and weight of these hypotheses. Some of the hypotheses are simple or technical, but a couple of the hypotheses are strong, and we would not expect them to be satisfied in the general case.

In Section 5, we consider Equation (E) with the additional hypothesis that it is a singularly perturbed equation. We will show that a class of singularly perturbed equations (Liénard equations) satisfy the assumptions introduced in Sections 2 and 3 and hence have solutions which describe the entrainment of frequency first obtained by van der Pol and van der Mark. The proof that the assumptions introduced in Sections 2 and 3 are satisfied is obtained by using a geometric construction in the phase space. Roughly speaking, this construction in the two-dimensional case is an annulus which contains the orbit of the periodic solution $\bar{x}(t)$ of Equation (E). This annulus has the property that every orbit of (E) which intersects the boundary of the annulus enters the annulus. (The annulus is an inflowing invariant manifold with boundary.) Such constructions have long been known for classes of two-dimensional 
autonomous systems (see Flanders and Stoker [13], LaSalle [18], and Stoker [33]). The construction has been extended to two-dimensional systems with large nonautonomous perturbations by Aboufadel [1], and we use a version of his result to show that the assumptions in Sections 2 and 3 are satisfied. In Section 6, the stability properties of the solutions are discussed.

The class of differential equations discussed in Section 5 is intended primarily as an example to illustrate application of the theory. It serves as a guide to further applications in that it shows that essentially all that is necessary for such applications is the construction of an annulus or, in higher dimensional cases, a tube with the properties described above. This is a considerably simpler procedure than to try to apply the general theory of singularly perturbed systems as described, e.g., in Mishchenko and Rozov [26] or the procedure used by N. Levinson [20]. The largest advantage of using such a construction is that direct study of the turning points is avoided. As shown in [26] and [20], the analytic approach to the study of solutions in the neighborhood of a turning point is complicated (to put it kindly). Moreover, if the geometric construction can be carried out, the sought-after solutions turn out to have desirable global stability properties. In a later paper, we will apply our theory to a four-dimensional model of the Purkinje fiber, in which there are two singular perturbation parameters, and indicate how the same kind of approach is suitable for other models of electrically active cells.

It should be pointed out that Cartwright and Littlewood $[4,5,6,21]$ made an extensive study of a nonlinear second-order equation which stemmed from work of van der Pol on radio circuit theory. In this remarkable series of papers, Cartwright and Littlewood studied a nonlinear equation with a forcing term for a wide range of values of several parameters. One of these parameters (the parameter $k$ ) is studied for large as well as small values. If $k$ is large, the equation can be rewritten as a singularly perturbed system. However, the singularly perturbed system that is obtained is of a more complicated form than the equation we study. The basic cause of this complication lies in the kind of discontinuous solution that must be used in the study. In our equations, the simple definition of discontinuous solution (see Mishchenko and Rozov [26]) can be used, but in the singularly perturbed system obtained from Cartwright and Littlewood if $k$ is large, it would be necessary to use the more complicated version of discontinuous solution developed by N. Levinson [20].

\section{Local nonautonomous perturbation of an autonomous system}

We consider the system

$$
\frac{d x}{d s}=F(x)
$$

where $x \in \mathbb{R}^{n}, F: \mathbb{R}^{n} \rightarrow \mathbb{R}^{n}$, and $F \in C^{3}\left(\mathbb{R}^{n}\right)$, and we assume that (1) has a solution $x_{0}(s)$ of period $T$. Let $G(s, \mu)$ be continuously differentiable on a bounded open set which contains $\mathbb{R} \times[-1,1]$ and $G(s, \mu)$ have as its range a subset of $\mathbb{R}^{n}$. Suppose also that $G$ has period $T(\mu)$ as a function of $s$, i.e., for all $s$,

$$
G(s+T(\mu), \mu)=G(s, \mu)
$$

where $T(\mu)$ is a continuously differentiable function of $\mu$ and $T(0)=T$. Assume also that $G(s, 0)=0$ for all $s$. The problem is to determine if, for sufficiently small $|\mu|$, the equation

$$
\frac{d x}{d s}=F(x)+G(s, \mu)
$$


has a solution of period $T(\mu)$ which is "near", in some sense, to the solution $x_{0}(s)$ of (1). In order to solve this problem, we apply the Poincaré method (see Roseau [31, Chapitre 18]).

First we reduce the equation to a problem involving only solutions of period $T$. Let

$$
t=\frac{s}{T(\mu)} T \quad \text { or } \quad s=\frac{T(\mu)}{T} t .
$$

Then

$$
G(s, \mu)=G\left[\frac{T(\mu)}{T} t, \mu\right],
$$

and, for each $\mu$, the function $G\left[\frac{T(\mu)}{T} t, \mu\right]$ has, as a function of $t$, the period $T$.

Rewriting (2) in terms of the variable $t$, we have

$$
\frac{d x}{d t}=\frac{d x}{d s} \frac{d s}{d t}=\frac{d x}{d s} \frac{T(\mu)}{T}=\frac{T(\mu)}{T}\left\{F(x)+G\left[\frac{T(\mu)}{T} t, \mu\right]\right\}
$$

or

$$
\frac{d x}{d t}=\frac{T(\mu)}{T}\left\{F(x)+G\left[\frac{T(\mu)}{T} t, \mu\right]\right\} .
$$

(Note that if $\mu=0,(3)$ becomes (1).) Denote $G\left[\frac{T(\mu)}{T} t, \mu\right]$ by $\tilde{g}(t, \mu)$. Then (3) becomes

$$
\frac{d x}{d t}=\frac{T(\mu)}{T}[F(x)+\tilde{g}(t, \mu)],
$$

and our question becomes, to determine if, for given $\mu$ with $|\mu|$ sufficiently small, (4) has a solution of period $T$ which is "near" the solution $x_{0}(s)$ of $(1)$.

Since $x_{0}(s)$ is a solution of $(1)$, then with a change of notation of the independent variable from $s$ to $t$, we may write

$$
\frac{d x_{0}}{d t}=F\left[x_{0}(t)\right]
$$

If $x(t)$ is a solution of $(4)$, let

$$
x(t)-x_{0}(t)=\mu u(t, \mu) .
$$

Subtracting (5) from (4), we have

$$
\mu \frac{d u}{d t}=F[x]-F\left[x_{0}\right]+\left[\frac{T(\mu)}{T}-1\right] F[x]+\frac{T(\mu)}{T} \tilde{g}(t, \mu) .
$$

Let the components of $F, x, u$ be

$$
\begin{aligned}
F & =\left(F_{1}, \ldots, F_{n}\right), \\
x & =\left(x_{1}, \ldots, x_{n}\right), \\
u & =\left(u_{1}, \ldots, u_{n}\right),
\end{aligned}
$$

and let $F_{x}(x)$ denote the matrix $\left[\frac{\partial F_{i}}{\partial x_{j}}(x)\right]$. Then we have

$$
\begin{aligned}
F[x] & -F\left[x_{0}\right]=F\left[x_{0}+\mu u\right]-F\left[x_{0}\right]=\left[F_{x}\left(x_{0}\right)\right][\mu u] \\
& +\left(\frac{\mu^{2}}{2 !}\left[\sum_{i, j=1}^{n}\left[\frac{\partial^{2} F_{1}}{\partial x_{i} \partial x_{j}}\left(x_{0}\right)\right]\left[u_{i}\right]\left[u_{j}\right]\right], \ldots, \frac{\mu^{2}}{2 !}\left[\sum_{i, j=1}^{n}\left[\frac{\partial^{2} F_{n}}{\partial x_{i} \partial x_{j}}\left(x_{0}\right)\left[u_{i}\right]\left[u_{j}\right]\right]\right)\right. \\
& +O\left(\mu^{3}\right),
\end{aligned}
$$


and, dividing (6) by $\mu$, we may write (6) as:

$$
\begin{aligned}
\frac{d u}{d t}=[ & \left.F_{x}\left(x_{0}\right)\right][u]+\mu \mathcal{R}_{2}(t, u) \mu^{2} \mathcal{R}_{3}(t, u, \mu) \\
& +\frac{1}{\mu}\left[\frac{T(\mu)}{T}-1\right] F\left(x_{0}+\mu u\right)+\frac{1}{\mu} \frac{T(\mu)}{T} \tilde{g}(t, \mu)
\end{aligned}
$$

where $\mathcal{R}_{2}$ has period $T$ as a function of $t$ and $\mu^{3} \mathcal{R}_{3}(t, u, \mu)$ is the remainder term denoted by $O\left(\mu^{3}\right)$ in (7). The expression $\mathcal{R}_{3}(t, u, \mu)$ also has period $T$ as a function of $t$.

Let $\mathcal{U}(t)$ be the fundamental matrix of

$$
\dot{u}=\left[F_{x}\left(x_{0}\right)\right] u
$$

such that $\mathcal{U}(0)=I$, the identity matrix, and let $u(t, \mu)$ be a solution of (8) with initial value

By the variation of constants formula,

$$
u(0, \mu)=u_{0}
$$

$$
\begin{aligned}
u(t, \mu)=[\mathcal{U}(t)] u_{0} & +\int_{0}^{t} \mathcal{U}(t-s)\left\{\mu \mathcal{R}_{2}(s, u)+\mu^{2} \mathcal{R}_{3}(s, u, \mu)\right. \\
& \left.+\frac{1}{\mu}\left[\frac{T(\mu)}{T}-1\right] F\left(x_{0}+\mu u\right)+\frac{1}{\mu} \frac{T(\mu)}{T} \tilde{g}(s, \mu)\right\} d s .
\end{aligned}
$$

A necessary and sufficient condition that $u(t, \mu)$ have period $T$ is

$$
u(T, \mu)-u(0, \mu)=u(T, \mu)-u_{0}=0 .
$$

Substituting from (10) into (11), we have

$$
\begin{aligned}
{[\mathcal{U}(T)-I] u_{0} } & +\int_{0}^{T} \mathcal{U}(T-s)\left\{\mu \mathcal{R}_{2}(s, u)+\mu^{2} \mathcal{R}_{3}(s, u, \mu)\right. \\
& \left.+\frac{1}{\mu}\left[\frac{T(\mu)}{T}-1\right] F\left(x_{0}+\mu u\right)+\frac{1}{\mu} \frac{T(\mu)}{T} \tilde{g}(s, \mu)\right\} d s=0 .
\end{aligned}
$$

In order to find a periodic solution $u(t, \mu)$, we must solve (12) for $u_{0}$ as a function of $\mu$.

From the hypotheses on $T(\mu)$, we may write

$$
\frac{T(\mu)}{T}-1=\frac{1}{T}[\mu k(\mu)]
$$

where $k(\mu)$ is a continuous function of $\mu$. By the hypotheses on $G(s, \mu)$, we may write

$$
\tilde{g}(t, \mu)=\mu \overline{\bar{g}}(t, \mu)
$$

where $\overline{\bar{g}}(t, \mu)$ is continuous in $(t, \mu)$ and has period $T$ as a function of $t$. Hence (12) can be written as

$$
\begin{aligned}
{[\mathcal{U}(T)-I] u_{0}+\int_{0}^{T} \mathcal{U}(} & -s)\left\{\mu \mathcal{R}_{2}(s, u)+\mu^{2} \mathcal{R}_{3}(s, u \mu)\right. \\
& \left.+\frac{k(\mu)}{T} F\left(x_{0}+\mu u\right)+\frac{T(\mu)}{T} \overline{\bar{g}}(s, \mu)\right\} d s=0 .
\end{aligned}
$$

Since $\mathcal{U}(0)=I$, the characteristic multipliers of $\left[F_{x}\left(x_{0}\right)\right]$ are the eigenvalues of the real constant matrix $C=\mathcal{U}(T)$. But the periodic function $\frac{d x_{0}}{d t}$ is a solution of (9). Hence $\left[F_{x}\left(x_{0}\right)\right]$ has at least one characteristic multiplier equal to 1 , i.e., $\mathcal{U}(T)$ has at least one eigenvalue equal to 1 . 
Assumption 1. The eigenvalue 1 of $\mathcal{U}(T)$ has algebraic multiplicity 1.

If we apply the classical procedure to solving (13) for $u_{0}$ as a function of $\mu$, it follows from Assumption 1 that the problem can be reduced to solving a single scalar bifurcation equation,

$$
B\left(u_{0}^{(1)}, \mu\right)=0
$$

where $u_{0}^{(1)}$ is a scalar component of $u_{0}$. Also the bifurcation equation with $\mu=0$, i.e.,

$$
B\left(u_{0}^{(1)}, 0\right)=0
$$

is quadratic in $u_{0}^{(1)}$. If this quadratic equation has two distinct real roots, it follows by application of the Implicit Function Theorem that (13) has two distinct real solutions $u_{0}$ for each $\mu$ such that $|\mu|$ is sufficiently small. If the quadratic equation has complex roots, then (13) has no real solutions $u_{0}$ if $|\mu|$ is sufficiently small. If the quadratic equation has a real root of multiplicity two, then a finer investigation must be undertaken.

The difficulty in applying these results to a specific case is that the computation of the coefficients in the quadratic equation may be practically difficult. To avoid this complication, we impose conditions on the growth of $T(\mu)$ and $\tilde{g}(t, \mu)$ which guarantee that the quadratic equation has a simple form. Since at this stage we are concerned only with the local problem $(|\mu|$ sufficiently small), these hypotheses do not present a complication.

Assumption 2. If $|\mu|$ is sufficiently small,

$$
T(\mu)-T=\mu^{3} h(\mu)
$$

where $h(\mu)$ is a differentiable function of $\mu$.

Assumption 3. If $|\mu|$ is sufficiently small,

$$
\tilde{g}(t, \mu)=\mu^{2} \bar{g}(t, \mu)
$$

where $\bar{g}$ has period $T$ as a function of $t$ and is differentiable in $(t, \mu)$.

Using Assumptions 2 and 3, we now make a detailed analysis of equation (8).

By Assumption 1, it follows that there exists a real constant nonsingular matrix $P$ such that

$$
P^{-1}\{\mathcal{U}(T)\} P=\left[\begin{array}{ll}
1 & 0 \\
0 & D
\end{array}\right]
$$

where $D$ is a real constant $(n-1) \times(n-1)$ matrix such that no eigenvalue of $D$ equals 1. Let $y_{0}=P^{-1} u_{0}$ or

$$
u_{0}=P y_{0}
$$

, and make the change of variables $u=P y$ in (8). Thus, (8) becomes, with Assumptions 2 and 3 ,

$$
\begin{gathered}
P \frac{d y}{d t}=\left[F_{x}\left(x_{0}\right)\right] P y+\mu \mathcal{R}_{2}(t, P y)+\mu^{2} \mathcal{R}_{3}(t, P y, \mu) \\
+T^{-1} \mu^{2} h(\mu) F\left(x_{0}+\mu P y\right)+\mu \bar{g}(t, \mu)
\end{gathered}
$$


Substituting $u_{0}=P_{y_{0}}$ into (12) and using Assumptions 2 and 3, we have

$$
\begin{aligned}
{[\mathcal{U}(T)-I] P y_{0}+} & \int_{0}^{T} \mathcal{U}(T-s)\left\{\mu \mathcal{R}_{2}(s, u)+\mu^{2} \mathcal{R}_{3}(s, u, \mu)\right. \\
& \left.+T^{-1} \mu^{2} h(\mu) F\left(x_{0}+\mu u\right)+\mu \frac{T(\mu)}{T} \bar{g}(s, \mu)\right\} d s=0
\end{aligned}
$$

where $u$ now denotes $P y\left(s, y_{0}, \mu\right)$ and $y\left(s, y_{0}, \mu\right)$ is the solution of (14) such that $y\left(0, y_{0}, \mu\right)=y_{0}$. Thus our problem has become that of solving (15) for $y_{0}$ as a function of $\mu$. Multiplying (15) on the left by $P^{-1}$, we obtain

$$
\left[P^{-1} \mathcal{U}(T) P-I\right] y_{0}+\int_{0}^{T} P^{-1} \mathcal{U}(T-s)\{\quad \cdots \quad\} d s=0
$$

or, from the definition of $P$, we have:

$$
\left[\begin{array}{cc}
0 & 0 \\
0 & D-I
\end{array}\right] y_{0}+\int_{0}^{T} P^{-1} \mathcal{U}(T-s)\{\quad \cdots \quad\} d s=0
$$

where $I$ now denotes the $(n-1) \times(n-1)$ identity matrix.

Now let

$$
y_{0}=\left[y_{1}, y_{2}, \ldots, y_{n}\right]^{T},
$$

and let $P_{n-1}$ be the projection of $\left[y_{1}, y_{2}, \ldots, y_{n}\right]^{T}$ into $\left[y_{2}, \ldots, y_{n}\right]^{T}$. An application of $P_{n-1}$ to (16) yields

$$
\begin{aligned}
(D-I)\left[\begin{array}{c}
y_{2} \\
\vdots \\
y_{n}
\end{array}\right]+P_{n-1} \int_{0}^{T} & P^{-1} \mathcal{U}(T-s)\left\{\mu \mathcal{R}_{2}(s, u)+\mu^{2} \mathcal{R}_{3}(s, u, \mu)\right. \\
& \left.+T^{-1} \mu^{2} h(\mu) F\left(x_{0}+\mu u\right)+\mu \frac{T(\mu)}{T} \bar{g}(s, \mu)\right\} d s=0 .
\end{aligned}
$$

At $\mu=0,(17)$ has the solution $\left[y_{2}^{0}, \ldots, y_{n}^{0}\right]^{T}=0$.

Lemma 1. There exist a neighborhood $N_{n-1}$ in $\mathbb{R}^{n-1}$ of $\left[y_{2}^{0}, \ldots, y_{n}^{0}\right]=0$, a neighborhood $N_{\mu}$ of $\mu=0$, and a bounded open set $F \subset \mathbb{R}$ such that if

$$
\left(y_{1}, \mu\right) \in F \times N_{\mu},
$$

then (17) has a unique solution $\left(y_{2}, \ldots, y_{n}\right)$ such that

$$
\left(y_{2}, \ldots, y_{n}\right) \in N_{n-1} \text {. }
$$

The solution $\left(y_{2}, \ldots, y_{n}\right)$ can be represented by

$$
\begin{gathered}
y_{2}=h_{2}\left(y_{1}, \mu\right), \\
\vdots \\
y_{n}=h_{n}\left(y_{1}, \mu\right)
\end{gathered}
$$

where $h_{j}\left(y_{1}, \mu\right), j=2, \ldots, n$, has $F \times N_{\mu}$ as its domain.

Proof. The proof is a straightforward application of the Implicit Function Theorem as follows. First, let $\bar{F}$ be the closure of a bounded open set $F$ in $\mathbb{R}$. Then there exists $\bar{\mu}>0$ and $N_{y}$, a bounded open neighborhood of $0 \in \mathbb{R}^{n-1}$ such that if $|\mu|<\bar{\mu}$ and $\left(y_{2}, \ldots, y_{n}\right) \in N_{y}$ and $y_{1} \in \bar{F}$, then, if

$$
y_{0}=\left(y_{1}, y_{2}, \ldots, y_{n}\right) \text {, }
$$


it follows that the solution $y\left(s, y_{0}, \mu\right)$ of (14) is defined for all $s \in[0, T]$. (This follows from the fact that $y\left(s, y_{0}, 0\right)$, as a solution of a linear homogeneous system with constant coefficients, is defined for all $s \in[0, T]$ and from the continuity in $\mu$ of $y\left(s, y_{0}, \mu\right)$.) Note that if $F$ and $N_{y}$ are selected first, then the bound $\bar{\mu}$ on $|\mu|$ may be taken independent of $y_{0} \in \bar{F}_{1} \times N_{y}$.

If $\mu=0, y_{j}=0$ for $j=2, \ldots, n$, and $\bar{y}_{1} \in \bar{F}$, then (17) is satisfied. That is, $\mu=0, y_{1}=\bar{y}_{1}, y_{j}=0(j=2, \ldots, n)$ is an initial solution of $(17)$. Since $(D-I)$ is nonsingular, it follows that the Implicit Function Theorem can be applied to solve for $y_{2}, \ldots, y_{n}$ in terms of $y_{1}$ and $\mu$ near this initial solution. This completes the proof of Lemma 1.

Remark. Note that for all $y_{1} \in F$, we have

$$
h_{j}\left(y_{1}, 0\right)=0 \quad(j=2, \ldots, n) .
$$

Now to solve (12) or, equivalently, (16), we solve (17) as described in Lemma 1 and substitute that solution into the first equation of (16). If we let $P_{1}$ be the projection of $\left(y_{1} \ldots, y_{n}\right)$ into $y_{1}$, then the first equation of $(16)$ is

$$
\begin{aligned}
P_{1} \int_{0}^{T} P^{-1} \mathcal{U}(T-s)\left\{\mu \mathcal{R}_{2}(s, u)+\mu^{2} \mathcal{R}_{3}(s, u, \mu)\right. \\
\left.+T^{-1} \mu^{2} h(\mu) F\left(x_{0}+\mu u\right)+\mu \frac{T(\mu)}{T} \bar{g}(s, \mu)\right\} d s=0
\end{aligned}
$$

where, as noted before, $u$ denotes $P y\left(t, y_{0}, \mu\right)$ and $y\left(t, y_{0}, \mu\right)$ is the solution of (14) such that $y\left(0, y_{0}, \mu\right)=y_{0}$.

Substituting the solution obtained in Lemma 1 means that in (18), $y_{0}$ becomes

$$
\left(y_{1}, h_{2}\left(y_{1}, \mu\right), \ldots, h_{n}\left(y_{1}, \mu\right)\right) .
$$

Now we must solve (18) for $y_{1}$ as a function of $\mu$. Dividing (18) by $\mu$ and setting $\mu=0$ in the resulting equation, we have as the bifurcation equation with $\mu=0$

$$
P_{1} \int_{0}^{T} P^{-1} \mathcal{U}(T-s)\left\{\mathcal{R}_{2}(s, u)+\bar{g}(s, 0)\right\} d s=0
$$

where $u$ now denotes $P y\left(s, y_{0}, 0\right)$ and $P y\left(s, y_{0}, 0\right)$ is the solution of (9) such that

$$
P y\left(0, y_{0}, 0\right)=P\left[\begin{array}{c}
y_{1} \\
0 \\
\vdots \\
0
\end{array}\right]
$$

(since $h_{j}\left(y_{1}, 0\right)=0$ for $\left.j=2, \ldots, n\right)$. If $P=\left[p_{i j}\right]$, then

$$
P\left[\begin{array}{c}
y_{1} \\
0 \\
\vdots \\
0
\end{array}\right]=y_{1}\left[\begin{array}{c}
p_{11} \\
p_{21} \\
\vdots \\
p_{n 1}
\end{array}\right] .
$$

Hence

$$
P y\left(s, y_{0}, 0\right)=y_{1} \mathcal{U}(s)\left[\begin{array}{c}
p_{11} \\
p_{21} \\
\vdots \\
p_{n 1}
\end{array}\right]
$$


Substituting this expression into (20) and using the form of $\mathcal{R}_{2}(s, u)$ given explicitly in $(7)$, we obtain

$$
A y_{1}^{2}+B=0
$$

where

$$
\begin{aligned}
A y_{1}^{2} & =P_{1} \int_{0}^{T} P^{-1} \mathcal{U}(T-s) \mathcal{R}_{2}\left(s, P y\left(s, y_{0}, 0\right)\right) d s \\
& =P_{1} \int_{0}^{T} P^{-1} \mathcal{U}(T-s) \mathcal{R}_{2}\left(s, y_{1} \mathcal{U}(s)\left[\begin{array}{c}
p_{11} \\
\vdots \\
p_{n 1}
\end{array}\right]\right) d s
\end{aligned}
$$

and

$$
B=P_{1} \int_{0}^{T} P^{-1} \mathcal{U}(T-s) \bar{g}(s, 0) d s .
$$

Assumption 4. $-\frac{B}{A}>0$.

From Assumption 4, it follows that (20) has two real nonzero solutions: $y_{1}=K$ and $y_{1}=-K$ where $K=\left(-\frac{B}{A}\right)^{1 / 2}$. Let $M_{0}$ denote the mapping from $\mathbb{R}$ into $\mathbb{R}$ defined by the left side of $(20)$, i.e.,

$$
M_{0}: y_{1} \rightarrow A y_{1}^{2}+B
$$

Then if $A>0[<0]$, it follows that

$$
\frac{d M_{0}}{d y_{1}}(K)>0 \quad[<0]
$$

and

$$
\frac{d M_{0}}{d y_{1}}(-K)<0 \quad[>0]
$$

Thus, $(K, 0)$ is an initial solution of (18) after (18) is divided by $\mu$, and we may apply the Implicit Function Theorem to solve for $y_{1}$ as a function of $\mu$ in a neighborhood of $(K, 0)$. A similar conclusion holds for $(-K, 0)$.

Summarizing the discussion in this section, we have:

Theorem 1. If Assumptions 1, 2, 3, 4 hold and if $|\mu|$ is sufficiently small, say $|\mu|<$ $\mu_{0}$, then (2) has two solutions $x(t, \mu)$ and $\bar{x}(t, \mu)$, each with period $T(\mu)$, where $x(t, \mu)$ and $\bar{x}(t, \mu)$ depend continuously on $\mu$ and

$$
\begin{aligned}
& \lim _{\mu \rightarrow 0} x(t, \mu)=x_{0}(t), \\
& \lim _{\mu \rightarrow 0} \bar{x}(t, \mu)=x_{0}(t)
\end{aligned}
$$

where the convergence is uniform for $t \in[0, T]$.

Conventional arguments show that if all the eigenvalues of $\mathcal{U}(T)$ which are different from 1 have absolute value less than 1 , then one of the periodic solutions given by Theorem 1 is asymptotically stable and the other is unstable. 
Remarks concerning Assumption 4. 1. Since equation (1) is autonomous, then $x_{0}(t+\delta)$, where $\delta$ is a real constant, is also a solution of (1) with period $T$. We have

$$
\frac{d x_{0}}{d t}(t+\delta)=F\left[x_{0}(t+\delta)\right]
$$

Letting

$$
x(t+\delta)=x_{0}(t+\delta)+\mu u(t+\delta, \mu),
$$

we seek a solution $x(t+\delta)$ of period $T$ of the equation

$$
\frac{d}{d t} x(t+\delta)=\frac{T(\mu)}{T}\left\{F[x(t+\delta)]+\mu^{2} \bar{g}(t, \mu)\right\} .
$$

Let $\tau=t+\delta$. Then equations (i) and (ii) become

$$
\frac{d x_{0}}{d \tau}(\tau)=F\left[x_{0}(\tau)\right]
$$

and

$$
\frac{d x}{d \tau}(\tau)=\frac{T(\mu)}{T}\left\{F[x(\tau)]+\mu^{2} \bar{g}(\tau-\delta, \mu)\right\} .
$$

Thus we have the same problem as earlier except that the phase of the forcing term is changed by $\delta$. The coefficient $A$ remains the same, but the sign of $B$ may be changed (e.g., if $\bar{g}(t, 0)=\sin t$, then if $\delta=\pi$, we have

$$
\bar{g}(t-\pi, 0)=\sin (t-\pi)=-\sin t=-\bar{g}(t, 0)) .
$$

Thus we may replace Assumption 4 by the following assumption. There exist real numbers $\delta_{1}$ and $\delta_{2}$ such that for $t \in[0, T]$,

$$
\bar{g}\left(t+\delta_{1}, 0\right)=-\bar{g}\left(t+\delta_{2}\right),
$$

or, more generally, coefficient $B$ has different signs for the two forcing terms $\bar{g}\left(t+\delta_{1}, 0\right)$ and $\bar{g}\left(t+\delta_{2}, 0\right)$.

2. If $A=0$ and $B \neq 0$, then if $|\mu|$ is sufficiently small, (18) has no solution $y_{1}$, and hence (2) has no periodic solutions of period $T(\mu)$ which are near $x_{0}(s)$. To proceed further with the analysis, it would be necessary to replace Assumption 2 by

$$
T(\mu)-T=\mu^{2} h(\mu) \text { or } \mu h(\mu)
$$

where $h(0) \neq 0$, or replace Assumption 3 by

$$
\tilde{g}(t, \mu)=\mu \bar{g}(t, \mu)
$$

where $\bar{g}(t, \mu) \neq 0$. Then the bifurcation equation with $\mu=0$ would be a more complicated equation than

$$
A y_{1}^{2}+B=0 .
$$

But the more complicated equation might have real solutions.

3. If $A=0$ and $B=0$, then (18) can be divided by $\mu^{2}$, and a finer higher-order investigation must be undertaken.

4. If $A \neq 0$ and $B=0$, then the procedure immediately before Theorem 1 breaks down because we obtain

$$
\frac{d M_{0}}{d y_{1}}(K)=\frac{d M_{0}}{d y_{1}}(-K)=0 .
$$

In this case, one reasonable course is to change $G(s, \mu)$ slightly so that $B \neq 0$. 


\section{Continuation of the perturbation}

In Section 2, it was shown that if $|\mu|$ is sufficiently small, then the equation

$$
\frac{d x}{d s}=F(x)+G(s, \mu)
$$

has a solution of period $T(\mu)$ which is near the given periodic solution $x_{0}(s)$ of

$$
\frac{d x}{d s}=F(x) \text {. }
$$

Our goal is to obtain a solution of period $T(\mu)$ for all $\mu \in[0,1]$. To obtain this result, we use

Assumption 5. There exists a positive constant $\mathcal{C}$ such that if $\mu \in[0,1]$ and $x(s, \mu)$ is a periodic solution of period $T(\mu)$ of $(2)$, then for $s \in[0, T(\mu)]$

$$
|x(s, \mu)| \leq \mathcal{C} .
$$

(In other words, there is an a priori bound on periodic solutions of (2) with $\mu \in[0,1]$.)

Lemma 2. Suppose Assumption 5 holds and suppose that a solution $x(t, \mu)$ of period $T$ of (4) is defined for

$$
\mu \in\left[0, \bar{\mu}_{1}\right)
$$

where $\bar{\mu}_{1}$ is a constant such that $\bar{\mu}_{1} \leq 1$, and $x(t, \mu)$ is continuous in $\mu$, uniformly for $t \in[0, T]$. Then

$$
\lim _{\mu \uparrow \bar{\mu}_{1}}[x(t, \mu) / t \in[0, T]]
$$

exists and is a solution $x(t, \mu)$ of period $T$ of

$$
\frac{d x}{d t}=\frac{T\left(\bar{\mu}_{1}\right)}{T}\left[F(x)+\tilde{g}\left(t, \bar{\mu}_{1}\right)\right]
$$

(Equation (21) is (4) with $\mu=\bar{\mu}_{1}$.)

Proof. First we use Assumption 5 to show that the set of periodic solutions $\{x(t, \mu) / \mu$ $\left.<\bar{\mu}_{1}\right\}$, given by hypothesis, converges uniformly on $[0, T]$ as $\mu \uparrow \bar{\mu}_{1}$ to a function $\tilde{x}(t)$. To show this, it is sufficient to prove that the set $\left\{x(t, \mu) / \mu<\bar{\mu}_{1}\right\}$ satisfies a Cauchy condition uniformly on $[0, T]$ as $\mu \uparrow \bar{\mu}_{1}$. That is, it is sufficient to prove that $\varepsilon>0$ implies there exists $\delta>0$ such that if

$$
\begin{aligned}
& 0<\bar{\mu}_{1}-\mu_{1}<\delta, \\
& 0<\bar{\mu}_{1}-\mu_{2}<\delta,
\end{aligned}
$$

and

$$
t \in[0, T]
$$

then

$$
\left|x\left(t, \mu_{2}\right)-x\left(t, \mu_{1}\right)\right|<\varepsilon .
$$

Using the notation introduced earlier (see (5a)), we have

$$
\begin{aligned}
\left|x\left(t, \mu_{2}\right)-x\left(t, \mu_{1}\right)\right| & =\left|x_{0}(t)+\mu_{2} u\left(t, \mu_{2}\right)-x_{0}(t)-\mu_{1} u\left(t, \mu_{1}\right)\right| \\
& =\left|\mu_{2} u\left(t, \mu_{2}\right)-\mu_{1} u\left(t, \mu_{1}\right)\right| \\
& \leq\left|\mu_{2} u\left(t, \mu_{2}\right)-\mu_{1} u\left(t, \mu_{2}\right)+\mu_{1} u\left(t, \mu_{2}\right)-\mu_{1} u\left(t, \mu_{1}\right)\right| \\
& \leq\left|\mu_{2}-\mu_{1}\right|\left|u\left(t, \mu_{2}\right)\right|+\mu_{1}\left|u\left(t, \mu_{2}\right)-u\left(t, \mu_{1}\right)\right| .
\end{aligned}
$$


From Assumption 5, it follows that there exists a constant $\mathcal{C}_{1}>0$ such that for all $\mu<\bar{\mu}_{1}$,

$$
\max _{t \in[0, T]}|u(t, \mu)|<\mathcal{C}_{1}
$$

Since $u(t, \mu)$ is continuous in $\mu$, uniformly for $t \in[0, T]$, then (22) yields the desired Cauchy condition, and therefore the set $\left\{x(x, \mu) / \mu<\bar{\mu}_{1}\right\}$ converges uniformly on $[0, T]$ as $\mu \uparrow \bar{\mu}_{1}$ to a function $\tilde{x}(t)$. Since the convergence is uniform in $t$, the function $\tilde{x}(t)$ is continuous for $t \in[0, T]$. Also the expression

$$
\frac{T(\mu)}{T}[F(x(t, \mu))+\tilde{g}(t, \mu)]
$$

which is the right-hand side of $(4)$ with $x=x(t, \mu)$, converges uniformly for $t \in[0, T]$ to

$$
\frac{T\left(\bar{\mu}_{1}\right)}{T}\left[F(\tilde{x}(t))+\tilde{g}\left(t, \bar{\mu}_{1}\right)\right] .
$$

But the left-hand side of (4) with $x=x(t, \mu)$ is

$$
\frac{d}{d t} x(t, \mu)
$$

Thus $\left\{\frac{d}{d t} x(t, \mu)\right\}$ converges to $\frac{d}{d t} \tilde{x}(t)$. Thus $\tilde{x}(t)$ is a solution of (21).

Finally, we must prove that $\tilde{x}(t)$ has period $T$. Since each $x(t, \mu)$ has period $T$, then for each $\mu$,

$$
x(0, \mu)=x(T, \mu)
$$

Hence

$$
\tilde{x}(0)=\tilde{x}(T) .
$$

Since $\tilde{g}\left(T, \mu_{1}\right)$ has period $T$ and since $\tilde{x}(t)$ is a solution of (21), then (23) implies that $\tilde{x}(t)$ has period $T$. This completes the proof of Lemma 2 .

Our next step is to show that a periodic solution can be obtained for $\mu>\bar{\mu}_{1}$. We consider

$$
\frac{d x}{d t}=\frac{T\left(\bar{\mu}_{1}+\delta^{2}\right)}{T}\left\{F(x)+\tilde{g}\left(t, \bar{\mu}_{1}+\delta^{2}\right)\right\}
$$

where $\delta \geq 0$. By Lemma 2 , if $\delta=0$ then (24) has the solution $x\left(t, \bar{\mu}_{1}\right)=\tilde{x}(t)$ of period $T$. That is,

$$
\frac{d x}{d t}\left(t, \bar{\mu}_{1}\right)=\frac{T\left(\bar{\mu}_{1}\right)}{T}\left\{F\left[x\left(t, \bar{\mu}_{1}\right)\right]+\tilde{g}\left(t, \bar{\mu}_{1}\right)\right\} .
$$

Suppose $x(t)$ is a solution of (24) and let

$$
x(t)=x\left(t, \bar{\mu}_{1}\right)+\delta u(t, \delta) .
$$

Substituting (26) in (24) and subtracting (25) from that result, we obtain (after division by $\delta$ )

$$
\frac{d u}{d t}=\left\{\frac{T\left(\bar{\mu}_{1}\right)}{T} F_{x}[\tilde{x}(t)]\right\} u+\delta[\cdots]
$$

where the terms in the brackets $[\cdots]$ are differentiable functions of $t, u, \delta$ and have period $T$ in the variable $t$. 
Lemma 3. If the characteristic multipliers of

$$
\frac{T\left(\bar{\mu}_{1}\right)}{T} F_{x}[\tilde{x}(t)]
$$

are all different from 1 , then there exists for sufficiently small $\delta>0$ a solution $u(t, \delta)$ of (27) of period $T$, and

$$
\lim _{\delta \downarrow 0} u(t, \delta)=0
$$

uniformly in $t \in[0, T]$.

Proof. Lemma 3 is a special case of the classical Poincaré theorem, see Coddington and Levinson [8, Chapter 14].

We impose the following assumption.

Assumption 6. The characteristic multipliers of the matrix

$$
\frac{T\left(\bar{\mu}_{1}\right)}{T} F_{x}[\tilde{x}(t)]
$$

are all different from 1.

From the derivation of (27) and Lemma 3, it follows that (2) has a solution of period $T(\mu)$ for $\mu \in\left(\bar{\mu}_{1}, \bar{\mu}_{1}+\delta_{0}\right)$ where $\delta_{0}$ is a (small) positive number.

Now we consider a hypothesis weaker than Assumption 6.

Assumption 6a. The matrix

$$
\frac{T\left(\bar{\mu}_{1}\right)}{T} F_{x}[\bar{x}(t)]
$$

has one characteristic multiplier equal to 1 and with algebraic multiplicity 1 . All the other characteristic multipliers are different from 1.

In this case we proceed as in the derivation of (15). We consider

$$
\frac{d x}{d t}=\frac{T\left(\bar{\mu}_{1}+\delta^{2}\right)}{T}\left\{F(x)+\tilde{g}\left(t, \bar{\mu}_{1}+\delta^{2}\right)\right\}
$$

where $\delta \geq 0$. If $\delta=0,(28)$ has the solution $x\left(t, \vec{\mu}_{1}\right)=\tilde{x}(t)$ of period $T$. That is,

$$
\frac{d}{d t} x\left(t, \bar{\mu}_{1}\right)=\frac{T\left(\bar{\mu}_{1}\right)}{T}\left\{F\left[x\left(t, \bar{\mu}_{1}\right)\right]+\tilde{g}\left(t, \bar{\mu}_{1}\right)\right\} .
$$

Suppose $x(t)$ is a solution of (28), and let

$$
x(t)=x\left(t, \bar{\mu}_{1}\right)+\delta u(t, \delta)=\tilde{x}(t)+\delta u(t, \delta) .
$$

Substituting (30) in (28) and subtracting (29) from that result, we obtain

$$
\begin{aligned}
\delta \frac{d u}{d t}= & \frac{T\left(\bar{\mu}_{1}+\delta^{2}\right)}{T} F[\tilde{x}+\delta u]-\frac{T\left(\bar{\mu}_{1}\right)}{T} F[\tilde{x}] \\
& +\left[\frac{T\left(\bar{\mu}_{1}\right)}{T} \tilde{g}_{\mu}\left(t, \bar{\mu}_{1}\right)+\frac{T^{\prime}\left(\bar{\mu}_{1}\right)}{T} \tilde{g}\left(t, \bar{\mu}_{1}\right)\right] \delta^{2}+O\left(\delta^{3}\right) \\
= & \frac{T\left(\bar{\mu}_{1}\right)}{T}\left[F_{x}(\tilde{x})\right] \delta u+\frac{T^{\prime}\left(\mu_{1}\right)}{T} F[\tilde{x}] \delta^{2}+\frac{T\left(\bar{\mu}_{1}\right)}{T} \\
& \times\left(\frac{1}{2} \sum_{i, j=1}^{n}\left[\frac{\partial^{2} F_{1}}{\partial x_{i} \partial x_{j}}(\tilde{x})\right] u_{i} u_{j}, \ldots, \frac{1}{2} \sum_{i, j=1}^{n}\left[\frac{\partial^{2} F_{n}}{\partial x_{i} \partial x_{j}}(\tilde{x})\right] u_{i} u_{j}\right) \delta^{2} \\
& +\left[\frac{T\left(\bar{\mu}_{1}\right)}{T} \tilde{g}_{\mu}\left(t, \bar{\mu}_{1}\right)+\frac{T^{\prime}\left(\bar{\mu}_{1}\right)}{T} \tilde{g}\left(t, \bar{\mu}_{1}\right)\right] \delta^{2}+O\left(\delta^{3}\right),
\end{aligned}
$$


or, dividing by $\delta$, we obtain

$$
\begin{aligned}
\frac{d u}{d t}= & \frac{T\left(\bar{\mu}_{1}\right)}{T} F_{x}[\tilde{x}] u+\delta \frac{T\left(\bar{\mu}_{1}\right)}{2 T}\left(\sum_{i, j=1}^{n}\left[\frac{\partial^{2} F_{1}(\tilde{x})}{\partial x_{i} \partial x_{j}}\right] u_{i} u_{j}, \ldots, \sum_{i, j=1}^{n}\left[\frac{\partial^{2} F_{n}(\tilde{x})}{\partial x_{i} \partial x_{j}}\right] u_{i} u_{j}\right) \\
& +\delta(H(t))+O\left(\delta^{2}\right)
\end{aligned}
$$

where

$$
\begin{gathered}
H(t)=\frac{T^{\prime}\left(\bar{\mu}_{1}\right)}{T} F[\tilde{x}]+\frac{T\left(\bar{\mu}_{1}\right)}{T} \tilde{g}_{\mu}\left(t, \bar{\mu}_{1}\right)+\frac{T^{\prime}\left(\bar{\mu}_{1}\right)}{T} \tilde{g}\left(t, \bar{\mu}_{1}\right), \\
O\left(\delta^{2}\right)=\delta^{2} K(\delta, t, u),
\end{gathered}
$$

$H(t)$ has period $T, K(\delta, t, u)$ has, as a function of $t$, period $T$, and $K$ is also a function of $u$.

Let $\mathcal{V}(t)$ be the fundamental matrix of

$$
\dot{u}=\frac{T\left(\bar{\mu}_{1}\right)}{T}\left[F_{x}(\tilde{x}(t))\right] u
$$

such that $\mathcal{V}(0)=I$, the identity matrix, and let $u(t, \mu)$ be a solution of (31) with initial value

$$
u(0, \mu)=u_{0}
$$

For convenience, denote

$$
\frac{T\left(\bar{\mu}_{1}\right)}{2 T}\left(\sum_{i, j=1}^{n}\left[\frac{\partial^{2} F_{1}(\tilde{x})}{\partial x_{i} \partial x_{j}}\right] u_{i} u_{j}, \ldots, \sum_{i, j=1}^{n}\left[\frac{\partial^{2} F_{n}(\tilde{x})}{\partial x_{i} \partial x_{j}}\right] u_{i} u_{j}\right)
$$

by $\mathcal{Q}_{2}(t, u)$.

By the variation of constants formula,

$$
u(t, \mu)=\mathcal{V}(t) u_{0}+\int_{0}^{t} \mathcal{V}(t-s)\left\{\delta \mathcal{Q}_{2}(s, u)+\delta H(s)+\delta^{2} K(\delta, s, u)\right\} d s .
$$

A necessary and sufficient condition that $u(t, \mu)$ have period $T$ is

$$
u(T, \mu)-u(0, \mu)=u(T, \mu)-u_{0}=0 .
$$

Substituting from (32) into (33), we have

$$
(\mathcal{V}(T)-I) u_{0}+\int_{0}^{T} \mathcal{V}(T-s)\left\{\delta \mathcal{Q}_{2}(s, u)+\delta H(s)+\delta^{2} K(\delta, s, u)\right\} d s=0
$$

By Assumption 6a, there exists a real nonsingular matrix $\bar{P}$ such that

$$
\bar{P}^{-1}\{\mathcal{V}(T)\} \bar{P}=\left[\begin{array}{ll}
1 & 0 \\
0 & \mathcal{D}
\end{array}\right]
$$

where $\mathcal{D}$ is a real constant $(n-1) \times(n-1)$ matrix such that no eigenvalue of $\mathcal{D}$ equals 1.

Let $y_{0}=\bar{P}^{-1} u_{0}$ or

$$
u_{0}=\bar{P} y_{0}
$$

and make the change of variables $u=\bar{P} y$ in (31). Then (31) becomes

$$
\bar{P} \frac{d y}{d t}=\frac{T\left(\bar{\mu}_{1}\right)}{T} F_{x}[\tilde{x}] \bar{P} y+\delta \mathcal{Q}_{2}(t, \bar{P} y)+\delta H(t)+\delta^{2} K(\delta, t, \bar{P} y) .
$$


Substituting from (35) into (33a) and multiplying on the left by $\bar{P}^{-1}$, we have

$$
\left[\bar{P}^{-1} \mathcal{V}(T) \bar{P}-I\right] y_{0}+\int_{0}^{T} \bar{P}^{-1} \mathcal{V}(T-s)\left\{\delta \mathcal{Q}_{2}(s, u)+\delta H(s)+\delta^{2} K(\delta, s, u)\right\} d s=0
$$

where $u$ now denotes $\bar{P} y\left(t, y_{0}, \delta\right)$ and $y\left(s, y_{0}, \mu\right)$ is the solution of (36) such that $y\left(0, y_{0}, \delta\right)=y_{0}$. Thus our problem becomes that of solving (37) for $y_{0}$ as a function of $\mu$. Applying (34) to (37), we have

$$
\left[\begin{array}{cc}
0 & 0 \\
0 & \mathcal{D}-I
\end{array}\right] y_{0}+\int_{0}^{T} \bar{P}^{-1} \mathcal{V}(T-s)\{\cdots\} d s=0 .
$$

Now in a manner exactly paralleling Lemma 1 , we may solve the last $(n-1)$ equations of $(37 \mathrm{a})$ to solve for $y_{2}, \ldots, y_{n}$ in terms of $y_{1}, \delta$ for $\delta$ in a neighborhood of $\delta=0$ and $y_{1}$ in a bounded open set of real numbers. We obtain

$$
y_{j}=h_{j}\left(y_{1}, \delta\right), \quad j=2, \ldots, n,
$$

and

$$
h_{j}\left(y_{1}, 0\right)=0, \quad j=2, \ldots, n .
$$

Substituting (38) into the first equation of (37a) and dividing by $\delta$, we have

$$
P_{1} \int_{0}^{T} \bar{P}^{-1} \mathcal{V}(T-s)\left\{\mathcal{Q}_{2}(s, u)+H(s)+\delta K(\delta, s, u)\right\} d s=0
$$

where $P_{1}$ is, as earlier, the projection of $\left(y_{1}, \ldots, y_{n}\right)$ into $y_{1}$. Now let $\delta=0$ in (39). Then

$$
u=\bar{P} y\left(s, y_{0}, 0\right)
$$

where $y\left(t, y_{0}, 0\right)$ is the solution of $(36)$ with $\delta=0$. That is, $y\left(t, y_{0}, 0\right)$ is the solution of

$$
\bar{P} \frac{d y}{d t}=\frac{T\left(\bar{\mu}_{1}\right)}{T} F_{x}[\tilde{x}] \bar{P} y .
$$

By $(38)$, since $\delta=0$,

$$
y_{0}=\left[\begin{array}{c}
y_{1} \\
0 \\
\vdots \\
0
\end{array}\right]
$$

Thus $\bar{P}\left[y\left(t, y_{0}, 0\right)\right]$ is the solution of

$$
\frac{d u}{d t}=\left\{\left[\frac{T\left(\bar{\mu}_{1}\right)}{T}\right] F_{x}(\tilde{x})\right\} u
$$

such that

$$
\bar{P} y\left(0, y_{0}, 0\right)=\bar{P}\left[\begin{array}{c}
y_{1} \\
0 \\
\vdots \\
0
\end{array}\right]
$$


If $\bar{P}=\left[p_{i j}\right]$, then

$$
\bar{P}\left[\begin{array}{c}
y_{1} \\
0 \\
\vdots \\
0
\end{array}\right]=y_{1}\left[\begin{array}{c}
\bar{p}_{11} \\
\bar{p}_{21} \\
\vdots \\
\bar{p}_{n 1}
\end{array}\right]
$$

Hence

$$
\bar{P} y\left(s, y_{0}, 0\right)=y_{1} \mathcal{V}(s)\left[\begin{array}{c}
\bar{p}_{11} \\
\bar{p}_{21} \\
\vdots \\
\bar{p}_{n 1}
\end{array}\right]
$$

Substituting from (40) into (39) with $\delta=0$, we obtain

$$
\bar{A} y_{1}^{2}+\bar{B}=0 \text {. }
$$

Assumption 6b. $-\frac{\bar{B}}{\bar{A}}>0$.

From Assumption $6 \mathrm{~b}$, it follows that (39) with $\delta=0$ has two real nonzero solutions. Hence, by the same arguments used for the proof of Theorem 1, we obtain

Lemma 4. If $\delta>0$ is sufficiently small, then (28) has two solutions of period $T$ which depend continuously on $\delta$ and converge as $\delta \rightarrow 0$ (uniformly in $t \in[0, T]$ ) to $x\left(t, \bar{\mu}_{1}\right)=\tilde{x}(t)$.

Inspection of (39) and (40) shows that

$$
\bar{A}=P_{1} \int_{0}^{T} \bar{P}^{-1} \mathcal{V}(T-s) \mathcal{Q}_{2}\left(s, y_{1} \mathcal{V}(s)\left[\begin{array}{c}
\bar{p}_{11} \\
\bar{p}_{21} \\
\vdots \\
\bar{p}_{n 1}
\end{array}\right]\right) d s
$$

and

$$
\bar{B}=P_{1} \int_{0}^{T} \bar{P}^{-1} \mathcal{V}(T-s) H(s) d s
$$

where $H(t)$ is defined in (31a).

Now we obtain the desired continuation.

Theorem 2. If Assumptions 1, 2, 3, 4, 5 are satisfied and if Assumption 6 or Assumptions $6 \mathrm{a}$ and $6 \mathrm{~b}$ hold for all $\bar{\mu}_{1} \in\left(\mu_{0}, 1\right)$ then for each $\mu \in[0,1],(2)$ has a solution $x(s, \mu)$ of period $T(\mu)$, and $x(s, \mu)$ is continuous in $\mu$.

Proof. We will prove that for each $\mu \in[0,1],(4)$ has a solution $x(t, \mu)$ of period $T$. The proof of the theorem then follows at once from the derivation of (4).

Let $E$ be a subset of $[0,1]$ with the following properties:

(i) if $\mu \in E$, then (4) has a solution $x(t, \mu)$ of period $T$ and $x(t, \mu)$ is continuous in $\mu$;

(ii) $E$ is connected;

(iii) $0 \in E$; and

(iv) the set $E$ is maximal with respect to properties (i), (ii), and (iii).

Since Assumptions 1, 2, 3, 4 hold, then by Theorem 1, there exists such a set $E$. We prove Theorem 2 by showing that $E=[0,1]$. By Assumption 5 and Lemma 2, the set 
$E$ is a closed interval $[0, a]$. If $a=1$, we have the desired result. If $a<1$, then since Assumptions 6 or $6 \mathrm{a}$ and $6 \mathrm{~b}$ hold at $\mu_{1}=a$, it follows by Lemmas 3 and 4 that $a$ is not the least upper bound of $D$. Thus we have a contradiction.

\section{Discussion of assumptions and result}

It should be pointed out first that Theorem 2 does not entirely answer the problem posed for Equation (2) at the beginning of Section 2. The reason is that we have not shown that the periodic solutions $x(s, \mu)$ obtained in Theorem 2 are in any sense close to the periodic solution $x_{0}(s)$ of (1). The proof of Theorem 1 shows that if $\mu$ is sufficiently small then $x(s, \mu)$ is close to $x_{0}(s)$. (More precisely, the periodic solution $x(t, \mu)$ of $(4)$ is uniformly close to $x_{0}(t)$.) However, there is no reason to conclude from our analysis, or in general, that all the solutions $x(t, \mu)$, where $\mu \in[0,1]$, are in any sense close together. As we will see in Section 5, such closeness of solutions does hold if (1) is a singularly perturbed system.

Assumptions 1 through 6 vary considerably in strength and seriousness. Assumption 1 is a typical assumption of nondegeneracy. Without Assumption 1, we would have several bifurcation equations instead of just one. Thus, the analysis would be more complicated. It also should be noted that in applications, it is natural to require that the solution $x_{0}(s)$ of the unperturbed (1) be phase asymptotically stable, i.e., to require the following stronger version of Assumption 1: $\mathcal{U}(T)$ has eigenvalue 1 with algebraic multiplicity 1 and all the remaining eigenvalues have absolute value less than 1. The significance of Assumptions 2 and 3 already has been discussed. The significance of Assumption 4 is discussed in the Remarks following Theorem 1.

Assumption 5 is an a priori bound on periodic solutions. The question of a priori bounds arises widely and naturally in nonlinear in-the-large problems. In particular, it arises in continuation procedures and consequently is not unexpected here.

Assumption 6 presents, in general, serious complications, because it requires that a fairly complicated condition on possible periodic solutions be satisfied. It is actually a complicated a priori condition on periodic solutions. Similar remarks hold for Assumptions 6a and 6b. However, in the singularly perturbed equations we will consider in Section 5, all the periodic solutons are close together. So the investigation of Assumptions 6, 6a, 6b is considerably less daunting.

\section{An example}

We consider a singularly perturbed system which is a special case of (1). The system to be studied is the Liénard equation

$$
\begin{aligned}
& \frac{d x}{d s}=\frac{1}{\varepsilon}[y-f(x)], \\
& \frac{d y}{d s}=-x
\end{aligned}
$$

where $\varepsilon$ is a small positive parameter. We assume that $f(x)$ is defined and has a continuous second derivative for all $x$, and that

$$
y=f(x)
$$

describes a smooth $s$-shaped curve as sketched in Figure 1. More precisely, we assume that there exist positive numbers $\alpha, \beta$ such that

1) if $x<-\alpha, f^{\prime}(x)>0$;

2) $f^{\prime}(-\alpha)=0, f(-\alpha)>0$; 


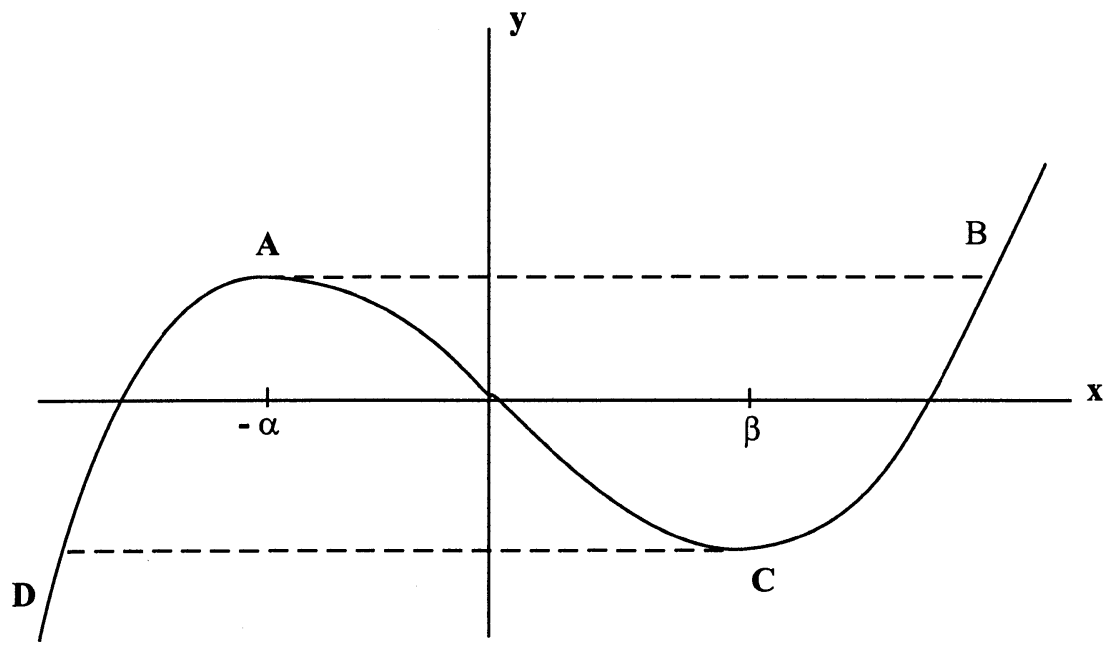

Figure 1

3) if $-\alpha<x<\beta, f^{\prime}(x)<0$;

4) if $-\alpha<x<0, f^{\prime \prime}(x)<0$;

5) $f^{\prime \prime}(0)=0 ; f(0)=0$

6) if $0<x<\beta, f^{\prime \prime}(x)>0$;

7) $f^{\prime}(\beta)=0 ; f(\beta)<0$; and

8) if $x>\beta, f^{\prime}(x)>0$.

(The hypotheses on $f$ are chosen for clarity and simplicity. They could be weakened but at the cost of additional uninteresting detail.)

The dashed closed curve ABCD in Figure 1 is called a closed discontinuous solution of (41).

We study (41) and the perturbed equation

$$
\begin{aligned}
& \frac{d x}{d s}=\frac{1}{\varepsilon}[y-f(x)]+G(s, \mu), \\
& \frac{d y}{d s}=-x
\end{aligned}
$$

where $\mu \in[0,1], G(s, \mu)$ is differentiable, $G(s, 0)=0$ and $G(s, \mu)$ has, as a function of $s$, the period $T(\mu)$, a differentiable function of $\mu$. We use the following results concerning Equations (41) and (42).

Theorem 3. If $\varepsilon_{0}>0$ is sufficiently small, then there exists an annulus $\mathfrak{A}\left(\varepsilon_{0}\right)$, bounded by two simple closed curves, which contains the discontinuous solution ABCD and which has the following five properties:

1) If the orbit of a solution of (42), with $0<\varepsilon \leq \varepsilon_{0}$ and $\mu \in[0,1]$, intersects the boundary of $\mathfrak{A}\left(\varepsilon_{0}\right)$, the orbit crosses the boundary of $\mathfrak{A}\left(\varepsilon_{0}\right)$ and enters $\mathfrak{A}\left(\varepsilon_{0}\right)$ (see Figure 2). Thus no orbit "escapes" $\mathfrak{A}\left(\varepsilon_{0}\right)$.

2) There exists a sequence $\left\{\varepsilon_{0}^{(n)}\right\}$ such that

$$
\lim _{n \rightarrow \infty} \varepsilon_{0}^{(n)}=0
$$

and

$$
\bigcap_{n} \mathfrak{A}\left(\varepsilon_{0}^{(n)}\right)=\mathrm{ABCD}
$$




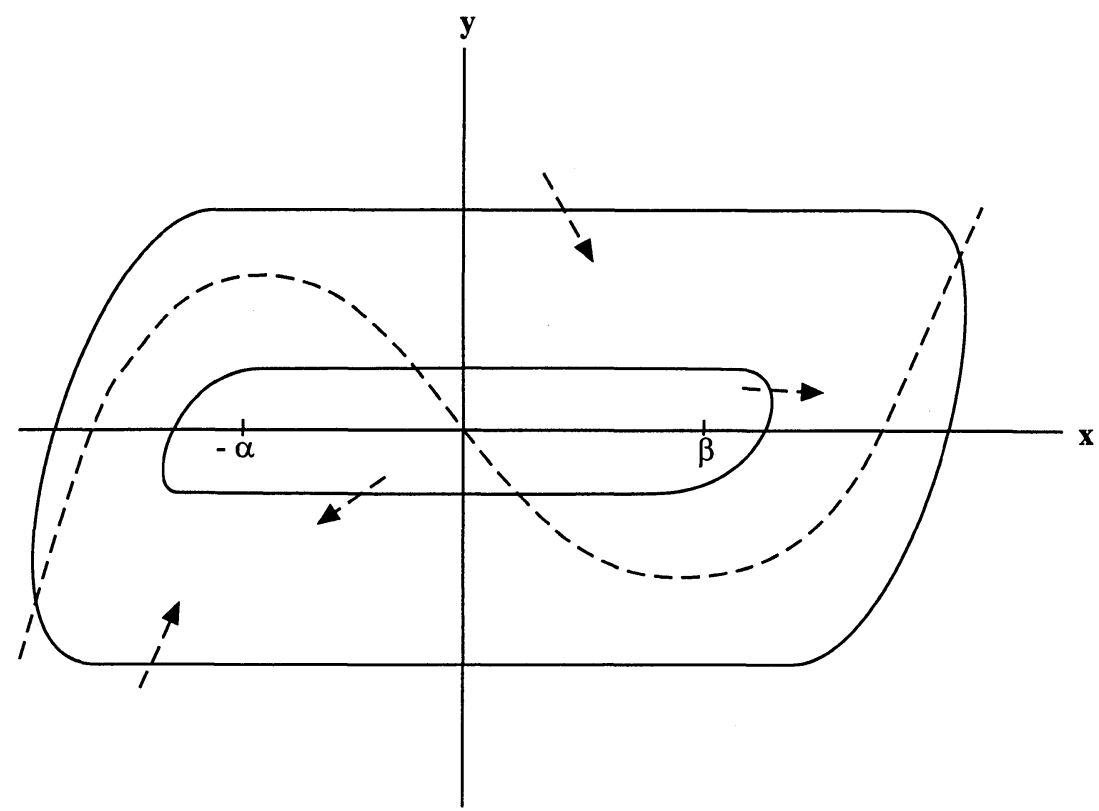

Figure 2

3) Let $\varepsilon>0$ be sufficiently small and fixed. Suppose the boundary of $\mathfrak{A}(\varepsilon)$ consists of the two simple closed curves $B_{i}$ and $B_{o}$ where $B_{i}$ is contained in the interior of $B_{o}$. Let Int $B_{j}$, Ext $B_{j}$ denote the interior and exterior, respectively, of $B_{j}$ for $j=o, i$. Then every solution outside $B_{o}$ approaches and enters $\mathfrak{A}(\varepsilon)$. More precisely, if $\left(x_{0}, y_{0}\right)$ is a point in Ext $B_{o}$ and if the solution $(x(t), y(t))$ is such that

$$
\left(x\left(t_{0}\right), y\left(t_{1}\right)\right)=\left(x_{0}, y_{0}\right),
$$

then there exists $t_{1}>t_{0}$ such that

$$
\left(x\left(t_{1}\right), y\left(t_{0}\right)\right) \in \mathfrak{A}(\varepsilon) .
$$

4) Given $m>0$, then there exists $\varepsilon_{0}>0$ and a neighborhood $N_{m}$ of $(0,0)$ such that:

a) the measure of $N_{m}$ is less than $m$ and $N_{m} \subset \operatorname{Int}\left(B_{i}\right)$,

b) if an orbit of (42) with $\varepsilon \leq \varepsilon_{0}$ passes through a point in

$$
\text { Int } B_{i}-N_{m} \text {, }
$$

then the orbit approaches and enters $\mathfrak{A}\left(\varepsilon_{0}\right)$. (As is shown in the proof, there is a periodic solution whose orbit is contained in $N_{m}$, but we will not be concerned with this periodic solution.)

5) Every orbit which passes through a point in

$$
\left(\text { Ext } B_{0} \cup \operatorname{Int} B_{i}\right)-N_{m}
$$

approaches and enters $\mathfrak{A}(\varepsilon)$. That is, most orbits enter $\mathfrak{A}(\varepsilon)$.

A proof of Theorem 3 is given in the Appendix.

The prototype of Theorem 3 was obtained by Flanders and Stoker [33] for the special case in which $\mu=0$ and (41) is the van der Pol equation. (See also LaSalle [18] and Stoker [33]. These results [13], [18], [33] are all for autonomous systems. That is, there is no periodic perturbation term.) 
The nonautonomous case has been studied by Aboufadel [1]. Theorem 3 is a special case of Chapter 1 of [1]. Aboufadel considers systems in which the second equation is

$$
\frac{d y}{d t}=g(x)+e_{2}(t)
$$

where appropriate assumptions are imposed on $g(x)$ and $e_{2}(t)$ is periodic. Aboufadel's construction is based on an analytic description. The proof of Theorem 3 given in the Appendix, which can be adapted to deal with the more general case, is more geometric but less elegant than Aboufadel's proof.

For the case $\mu=0$, if $\varepsilon_{0}$ is sufficiently small, it follows by construction of a Poincaré map (see, e.g., Stoker [33, Appendices III and IV]) that if $0<\varepsilon \leq \varepsilon_{0}$, (41) has a periodic solution whose orbit is contained in $\mathfrak{A}\left(\varepsilon_{0}\right)$ and is close to the closed discontinuous solution. Let us denote that periodic solution by $\left(x_{\varepsilon}(s), y_{\varepsilon}(s)\right)$ and denote the period of $\left(x_{\varepsilon}(s), y_{\varepsilon}(s)\right)$ by $T$.

We consider the following problem: given a function $G(s)$ where $G(s)$ has period $T_{1} \neq T$, to show that if $0<\varepsilon \leq \varepsilon_{0}$, then the equation

$$
\begin{aligned}
& \frac{d x}{d s}=\frac{1}{\varepsilon}[y-f(x)]+G(s), \\
& \frac{d y}{d s}=-x
\end{aligned}
$$

has a solution of period $T_{1}$ which is in some sense close to the periodic solution $\left(x_{\varepsilon}(s), y_{\varepsilon}(s)\right)$. To solve this problem, we introduce the parameter $\mu \in[0,1]$, define

$$
T(\mu)=T+\mu^{3}\left(T_{1}-T\right),
$$

and consider the equation

$$
\begin{aligned}
& \frac{d x}{d s}=\frac{1}{\varepsilon}[y-f(x)]+\mu^{2} G\left(\frac{T_{1}}{T(\mu)} s\right), \\
& \frac{d y}{d s}=-x .
\end{aligned}
$$

(If $\mu=0,(44)$ is (41) and if $\mu=1,(44)$ is (43).) We make the transformation of variables

$$
t=\frac{s}{T(\mu)} T
$$

Then (44) becomes

$$
\begin{aligned}
& \frac{d x}{d t}=\frac{T(\mu)}{T}\left\{\frac{1}{\varepsilon}[y-f(x)]+\mu^{2} G\left(\frac{T_{1}}{T} t\right)\right\}, \\
& \frac{d y}{d t}=-\left[\frac{T(\mu)}{T}\right] x .
\end{aligned}
$$

We apply the theory of Sections 2 and 3 to Eq. (45). That is, we determine conditions under which Assumptions 1 through 6 (or $6 \mathrm{a}, 6 \mathrm{~b}$ ) are satisfied and thus show that (45) has a solution of period $T(\mu)$ for all $\mu \in[0,1]$.

First we note that if periodic solutions are obtained by applying Theorem 2 , then their orbits are all in $\mathfrak{A}(\varepsilon)$. More precisely, we have the

Observation. Suppose that

$$
\{(x(t, \mu), y(t, \mu)) / \mu \in[0,1]\}
$$

is a set of solutions that can be obtained by applying Theorem 2 . Then the orbit of each solution $(x(t, \mu), y(t, \mu))$ is contained in the interior of $\mathfrak{A}(\varepsilon)$. 
Proof. By Part 1) of Theorem 3, no orbit of a periodic solution of (42) can intersect the boundary of $\mathfrak{A}(\varepsilon)$. Let

$$
\tilde{\mu}=\operatorname{lub}_{\bar{\mu}}\left\{\begin{array}{l}
\text { initial value }(x(0, \mu), y(0, \mu)) \text { is contained } \\
\text { in the interior of } \mathfrak{A}(\varepsilon) \text { for } \mu \in[0, \bar{\mu}]
\end{array}\right\} .
$$

If $\tilde{\mu}<1$, then $(x(0, \tilde{\mu}), y(0, \tilde{\mu}))$ is a point on the boundary of $\mathfrak{A}(\varepsilon)$, a contradiction.

Assumption 5 follows from this observation.

From the definition of $T(\mu)$ above, it follows at once that Assumption 2 is satisfied with $h(\mu)=T_{1}-T$. Assumption 3 is satisfied with

$$
\bar{g}(t, \mu)=\left[\begin{array}{c}
G\left[\frac{T_{1}}{T} t\right] \\
0
\end{array}\right]
$$

All the remaining assumptions, i.e., Assumptions 1, 4, 6 or $6 \mathrm{a}$, and $6 \mathrm{~b}$, depend on showing that particular integrals are different from zero.

To check Assumption 1, we note that the expression $F_{x}\left[x_{0}(t)\right]$ introduced in Section 2 (just before (7)) becomes, in the case of (45), the matrix

$$
M(t)=\left[\begin{array}{cc}
-\frac{1}{\varepsilon} f^{\prime}\left[x_{\varepsilon}(t)\right] & \frac{1}{\varepsilon} \\
-1 & 0
\end{array}\right]
$$

where $x_{\varepsilon}(t)$ has period $T$. By a standard result (see Cesari [7] or Cronin [11]), the product of the characteristic multipliers is

$$
\begin{aligned}
\lambda_{1} \lambda_{2} & =\exp \left\{\int_{0}^{T}[\operatorname{tr} M(t)] d t\right\} \\
& =\exp \left\{\int_{0}^{T}\left[-\frac{1}{\varepsilon} f^{\prime}\left[x_{\varepsilon}(t)\right]\right] d t\right\} .
\end{aligned}
$$

Since $f^{\prime}\left[x_{\varepsilon}(t)\right]$ is real, then $\lambda_{1} \lambda_{2}$ is positive. Since $\left(\frac{d}{d t} x_{\varepsilon}(t), \frac{d}{d t} y_{\varepsilon}(t)\right)$ is a periodic solution of

$$
\left[\begin{array}{l}
x^{\prime} \\
y^{\prime}
\end{array}\right]=M(t)\left[\begin{array}{l}
x \\
y
\end{array}\right]
$$

then one of the characteristic multipliers, say $\lambda_{1}$, is equal to 1 . If

$$
\int_{0}^{T} f^{\prime}\left[x_{\varepsilon}(t)\right] d t \neq 0
$$

then Assumption 1 is satisfied. Indeed, if

$$
\int_{0}^{T} f^{\prime}\left[x_{\varepsilon}(t)\right] d t>0 \quad[<0]
$$

it follows that

$$
\lim _{\varepsilon \rightarrow 0} \lambda_{2}=0 \quad[=\infty]
$$

(Thus, it follows that if

$$
\int_{0}^{T} f^{\prime}\left[x_{\varepsilon}(t)\right] d t>0 \quad[<0],
$$

then the solution $\left(x_{\varepsilon}(t), y_{\varepsilon}(t)\right)$ is phase asymptotically stable [unstable].) 
Similarly, if Assumption 6 is not satisfied, then in order to show that Assumption $6 \mathrm{a}$ is satisfied, it is sufficient to show that if one characteristic multiplier of

$$
\frac{T\left(\mu_{1}\right)}{T} F_{x}\left[x\left(t, \mu_{1}\right)\right]
$$

is equal to 1 , the other characteristic multiplier is different from 1 . If one characteristic multiplier is equal to 1 , the other characteristic multiplier is equal to

$$
\exp \left\{\int_{0}^{T}\left\{-\frac{1}{\varepsilon} \frac{T\left(\mu_{1}\right)}{T} f^{\prime}\left[x_{1}\left(t, \mu_{1}\right)\right]\right\} d t\right\}
$$

(where $x_{1}\left(t, \mu_{1}\right)$ denotes now the first component of the periodic solution $x\left(t, \mu_{1}\right)$ ). Hence, the other characteristic multiplier is different from 1 if and only if

$$
\int_{0}^{T}\left\{f^{\prime}\left[x_{1}\left(t, \mu_{1}\right)\right]\right\} d t \neq 0
$$

i.e., if and only if the mean value of $f^{\prime}\left[x_{1}\left(t, \mu_{1}\right)\right]$ is nonzero.

Since $\left(x_{\varepsilon}(t), y_{\varepsilon}(t)\right)$ and $x\left(t, \mu_{1}\right)$ are in the annulus $\mathfrak{A}(\varepsilon)$, then by Part 2) of Theorem 3 , the orbits of $\left(x_{\varepsilon}(t), y_{\varepsilon}(t)\right)$ and $x\left(t, \mu_{1}\right)$ are very close to the discontinuous solution ABCD. Then the verification of conditions (47) and (48) may be simple. For example, if

$$
f(x)=\frac{x^{3}}{3}-x
$$

(i.e., (41) is the van der Pol equation), then (48) becomes

$$
\int_{0}^{T}\left\{1-\left[x_{1}\left(t, \mu_{1}\right)\right]^{2}\right\} d t \neq 0
$$

where $x_{1}\left(t, \mu_{1}\right)$ denotes the first component of $x\left(t, \mu_{1}\right)$. Then elementary arguments show that if $\mathfrak{A}(\varepsilon)$ is close enough to the discontinuous solution (more precisely, if the $\varepsilon_{n}, \delta_{n}$, and $m_{0}^{(n)}$, described in the proof of Part 2) of Theorem 3 are sufficiently small), then the integral in (49) is bounded away from zero uniformly in $\mu_{1} \in[0,1]$.

For verification of Assumption 4, we require first that

$$
A=P_{1} \int_{0}^{T} P^{-1} \mathcal{U}(T-s) \mathcal{R}_{2}\left[s, P y\left(s, y_{0}, 0\right)\right] d s \neq 0 .
$$

For our example, the matrix $\mathcal{U}(t)$ is the fundamental matrix of $(46)$ such that $\mathcal{U}(0)=I$, the identity matrix. If we write

$$
\mathcal{U}(s)=\left[\begin{array}{ll}
v_{11}(s) & v_{12}(s) \\
v_{21}(s) & v_{22}(s)
\end{array}\right]
$$

use (20), and the fact that

$$
P^{-1} \mathcal{U}(T)=\left[\begin{array}{cc}
1 & 0 \\
0 & \lambda_{2}
\end{array}\right] P^{-1}
$$

then (50) becomes, for our example

$$
A=P_{1} \int_{0}^{T}\left[\begin{array}{cc}
1 & 0 \\
0 & \lambda_{2}
\end{array}\right] P^{-1}[\mathcal{U}(s)]^{-1} V(s) d s \neq 0
$$

or

$$
A=P_{1} \int_{0}^{T}[\mathcal{U}(s) P]^{-1} V(s) d s \neq 0
$$


where

$$
V(s)=\left[\begin{array}{c}
\left\{\frac{f^{\prime \prime}}{2}\left[x_{\varepsilon}(s)\right]\right\}\left\{\left[p_{11} v_{11}(s)+p_{21} v_{12}(s)\right]^{2}\right\} \\
0
\end{array}\right]
$$

We require also that the number

$$
B=P_{1} \int_{0}^{T} P^{-1} \mathcal{U}(T-s) \bar{g}(s, 0) d s
$$

be nonzero and have sign opposite to that of $A$. For our example, we have

$$
B=P_{1} \int_{0}^{T} P^{-1} \mathcal{U}(T-s)\left[\begin{array}{c}
G\left(\frac{T_{1}}{T} s\right) \\
0
\end{array}\right] d s
$$

or

$$
\begin{aligned}
B & =P_{1} \int_{0}^{T}\left[\begin{array}{cc}
1 & 0 \\
0 & \lambda_{2}
\end{array}\right] P^{-1}[\mathcal{U}(s)]^{-1}\left[\begin{array}{c}
G\left(\frac{T_{1}}{T} s\right) \\
0
\end{array}\right] d s \\
& =P_{1} \int_{0}^{T} P^{-1}[\mathcal{U}(s)]^{-1}\left[\begin{array}{c}
G\left(\frac{T_{1}}{T} s\right) \\
0
\end{array}\right] d s .
\end{aligned}
$$

For the verification of Assumption 6b, we note that since the orbit of $x\left(t, \mu_{1}\right)$ is in $\mathfrak{A}(\varepsilon)$, then $x\left(t, \mu_{1}\right)$ is very close to the discontinuous solution. Hence, verification of Assumption $6 \mathrm{~b}$ in particular cases requires arguments similar to those used to verify Assumption 4.

\section{Stability of solutions in example}

Conventional arguments can be used to study the local stability properties of the periodic solutions obtained in Section 5. If

$$
\int_{0}^{T} f^{\prime}\left[x_{\varepsilon}(t)\right] d t>0
$$

then for $|\mu|$ sufficiently small, (45) has one asymptotically stable periodic solution and one unstable periodic solution. Similar arguments can be used for all $\mu \in[0,1]$. But, because of the very definition of stability, all the results obtained may be of questionable practical value (see [11, pp. 192-193]).

Far more important for applications is the approximate global asymptotic stability displayed by the solutions in Section 5 . Conditions 3), 4), and 5) of Theorem 3 show that periodic solutions have such approximate global asymptotic stability: since the measure of the neighborhood $N_{m}$ can be made as small as desired by choosing $\varepsilon$ sufficiently small, then we may say that if $\varepsilon$ is sufficiently small, most solutions enter the annulus $\mathfrak{A}(\varepsilon)$. Moreover, by Part 2) of Theorem 3 , if $\varepsilon$ is small, then $\mathfrak{A}(\varepsilon)$ "closes down" on the discontinuous solution ABCD.

\section{Appendix}

Proof of Theorem 3.

1) Construction of the annulus

For the construction we consider the curves (see Figure 3)

$$
\begin{aligned}
& y=f(x)+\delta, \\
& y=f(x), \\
& y=f(x)-\delta
\end{aligned}
$$


where $\delta>0$. Let $P_{1} P_{2}$ be the horizontal segment between the curves

$$
\begin{aligned}
& y=f(x)+\delta \\
& y=f(x)-\delta,
\end{aligned}
$$

as indicated in Figure 3. Let the $y$-coordinate of each point in $P_{1} P_{2}$ be $f(-\alpha)+d$ where $d>0$. Let

$$
P_{3}=(\beta, f(\beta)+\delta)
$$

and

$$
P_{4}=(0, f(\beta)+\delta)
$$

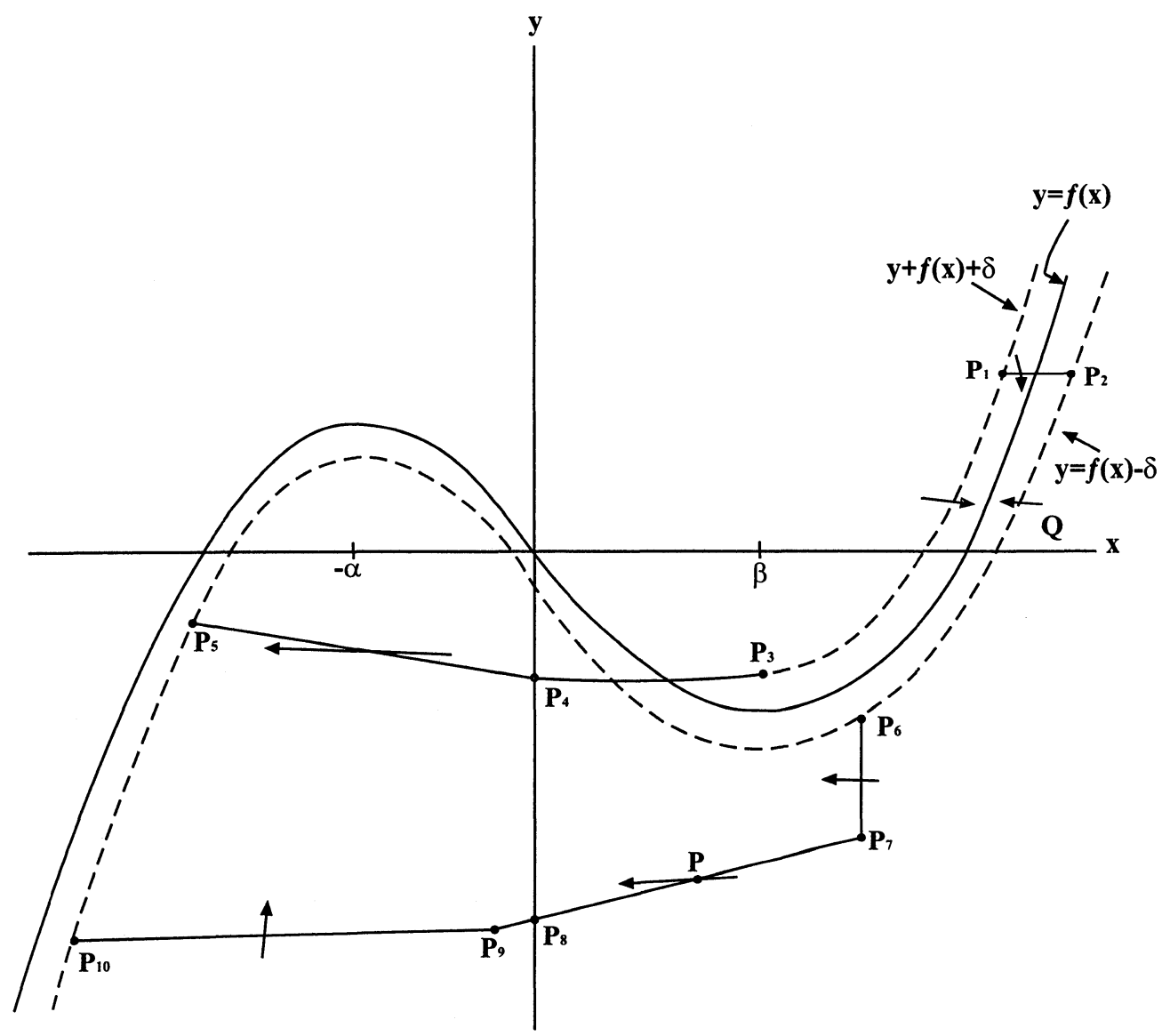

Figure 3

First, we choose $\delta>0$ small enough so that the point $P_{4}$ is below the intersection of the curve

$$
y=f(x)-\delta
$$

with the $y$-axis. More precisely, since

$$
P_{4}=(0, f(\beta)+\delta)
$$


and the intersection of the curve $(\mathcal{K})$ with the $y$-axis is $(0,-\delta)$, we choose $\delta$ small enough so that

$$
f(\beta)+\delta<-\delta
$$

or

$$
2 \delta<-f(\beta),
$$

The orbits cross $P_{1} P_{2}$ as indicated in Figure 3 because $\frac{d y}{d t}=-x$. If $\varepsilon$ is sufficiently small, then

$$
\frac{\delta}{\varepsilon}>\max _{(s, \mu)}|G(s, \mu)|
$$

and the orbits cross the curve $P_{1} P_{3}$ as sketched in Figure 3. Also the orbits cross the segment $P_{3} P_{4}$ as sketched because $\frac{d y}{d t}=-x$.

Now we choose the point $P_{5}$ so that

i) $P_{5}$ is on the curve $\mathcal{K}$;

ii) the $x$-coordinate of $P_{5}$ is less than $-\alpha$; and

iii) the $y$-coordinate of $P_{5}$ is a negative number $-\gamma$ such that

$$
-\gamma>f(\beta)+\delta
$$

where $f(\beta)+\delta$ is the $y$-coordinate of $P_{4}$.

Let $(\bar{x}, \bar{y})$ be a point on the line segment $P_{4} P_{5}$. If an orbit passes through $(\bar{x}, \bar{y})$, then the slope of the tangent at $(\bar{x}, \bar{y})$ of the orbit is

$$
\frac{-\bar{x}}{\frac{1}{\varepsilon}[\bar{y}-f(\bar{x})]+G\left(s_{0}, \mu\right)}=\frac{-\varepsilon \bar{x}}{[\bar{y}-f(\bar{x})]+\varepsilon G\left(s_{0}, \mu\right)}
$$

where $s_{0}$ is a fixed value. Since the line segment $P_{4} P_{5}$ is below or on the curve $\mathcal{K}$, i.e., the curve

$$
y=f(x)-\delta
$$

or

$$
y-f(x)=-\delta
$$

then

$$
\bar{y}-f(\bar{x}) \leq-\delta
$$

Let

$$
M=\max _{(s, \mu)}|G(s, \mu)| .
$$

Choose $\varepsilon>0$ small enough so that

$$
\varepsilon M<\frac{\delta}{2}
$$

Then for all $(s, \mu)$ and for all $(x, y) \in P_{4} P_{5}$

$$
|y-f(x)+\varepsilon G(s, \mu)| \geq \frac{\delta}{2} .
$$

Since $P_{4} P_{5}$ is a bounded set, then there exists $R>0$ such that if $(x, y) \in P_{4} P_{5}$, then

$$
|x| \leq R \text {. }
$$


Then it follows from (1) that the absolute value of the slope of the tangent at $(\bar{x}, \bar{y}) \in$ $P_{4} P_{5}$ of an orbit which passes through $(\bar{x}, \bar{y})$ is less than

$$
\frac{\varepsilon R}{\delta / 2}=\frac{2 \varepsilon R}{\delta}
$$

Let $m_{0}$ be the absolute value of the slope of the line determined by $P_{4} P_{5}$. If $\varepsilon$ is sufficiently small, then

$$
\frac{2 \varepsilon R}{\delta}<m_{0}
$$

Hence, the orbits cross $P_{4} P_{5}$ as sketched in Figure 3.

Now we choose $P_{6}=\left(x_{0}, y_{0}\right)$ on the curve $\mathcal{K}$ so that $x_{0}=\beta_{0}>\beta$ and so that

$$
y_{0}<f(\beta)-\frac{\delta}{2} \text {. }
$$

If $\varepsilon$ is sufficiently small, the orbits cross the curve $P_{2} P_{6}$ as indicated in Figure 3 . The reason for this is that the slope of the tangent to the point $Q$ on $P_{2} P_{6}$ has a positive lower bound, say $b$; and if an orbit intersects $P_{2} P_{6}$ at a point $Q$ and if $\varepsilon$ is sufficiently small, the absolute value of the slope of the tangent to the orbit at $Q$ is less than $b$.

Let $P_{7}=\left(x_{1}, y_{1}\right)$ be such that

$$
x_{1}=x_{0}=\beta_{0}
$$

and

$$
y_{1}<f(\beta)-\delta
$$

Then by similar arguments, the orbits cross $P_{6} P_{7}$ as indicated in Figure 3.

Now suppose $P=\left(x_{2}, y_{2}\right)$ is such that

$$
y_{2}<y_{1}<f(\beta)-\delta
$$

and

$$
x_{2} \in\left[0, \beta_{0}\right] .
$$

We consider the slope of the tangent at $P$ of an orbit which passes through $P$. The absolute value of that slope is

$$
\left|\frac{d y / d t}{d x / d t}\right|=\left|\frac{-x_{2}}{1 / \varepsilon\left[y_{2}-f\left(x_{2}\right)\right]+G\left(s_{0}, \mu\right)}\right| \leq \frac{\varepsilon \beta_{0}}{\left|y_{2}-f\left(x_{2}\right)+\varepsilon G\left(s_{0}, \mu\right)\right|}
$$

where $s_{0}$ is a fixed value. Next we have

$$
\begin{aligned}
\left|\left[y_{2}-f\left(x_{2}\right)\right]+\varepsilon G\left(s_{0}, \mu\right)\right| & \geq\left|y_{2}-f\left(x_{2}\right)\right|-\left|\varepsilon G\left(s_{0}, \mu\right)\right| \\
& \geq\left|y_{2}-f\left(x_{2}\right)\right|-\varepsilon M \\
& \geq\left|y_{2}-f\left(x_{2}\right)\right|-\frac{\delta}{2} .
\end{aligned}
$$

Since $x_{2} \in\left[0, \beta_{0}\right]$ and

$$
y_{2}<f(\beta)-\delta<0
$$

it follows that

$$
y_{2}-f\left(x_{2}\right)<-\delta,
$$

and hence

$$
\left|y_{2}-f\left(x_{2}\right)\right|>\delta
$$


Thus, from (3), we obtain

$$
\left|\left[y_{2}-f\left(x_{2}\right)\right]+\varepsilon G\left(s_{0}, \mu\right)\right| \geq \frac{\delta}{2}
$$

and (2) yields

$$
\left|\frac{d y / d t}{d x / d t}\right| \leq \frac{\varepsilon \beta_{0}}{\delta / 2}=\frac{2 \beta_{0} \varepsilon}{\delta} .
$$

Thus, we have shown that given $m_{0}>0$, then if $\varepsilon$ is sufficiently small,

$$
\left|\frac{d y / d t}{d x / d t}\right|<\frac{m_{0}}{2} .
$$

Let $P_{8}$ be chosen so that $P_{8}$ is on the $y$-axis and $P_{7} P_{8}$ is contained in a line with slope $m_{0}$. Then the orbits cross $P_{7} P_{8}$ as sketched in Figure 3 . The orbits similarly will cross $P_{7} P_{9}$ where $P_{7} P_{9}$ is an extensionof $P_{7} P_{8}$ as shown in Figure 3.

Finally, let $P_{9} P_{10}$ where $P_{10}$ is a point on curve $\mathcal{K}$, be a horizontal line segment. Since

$$
\frac{d y}{d t}=-x
$$

then the orbits cross $P_{9} P_{10}$ as indicated in Figure 3.

To complete the construction of the annulus, we proceed by symmetrical arguments to obtain the annulus sketched in Figure 4.

In Figure 4, the simple closed curve $B_{o}$ is

$$
P_{1} P_{2} P_{6} P_{7} P_{9} P_{10} P_{2}^{\prime} P_{6}^{\prime} P_{7}^{\prime} P_{9}^{\prime} P_{1},
$$

and the simple closed curve $B_{i}$ is

$$
P_{5}^{\prime} P_{3} P_{4} P_{5} P_{3}^{\prime} P_{4}^{\prime} P_{5}^{\prime} .
$$

Proof of 2): Let $\left\{\delta_{n}\right\}$ be a monotonic decreasing sequence such that $\delta_{n}>0$ and $\lim _{n \rightarrow \infty} \delta_{n}=0$. Let $\left\{m_{0}^{(n)}\right\}$ be a monotonic decreasing sequence such that $m_{0}^{(n)}>0$ and $\lim _{n \rightarrow \infty} m_{0}^{(n)}=0$. Let $\varepsilon_{n}$ be the $\varepsilon$ in the construction of the annulus if $\delta=\delta_{n}$ and $m_{0}=m_{0}^{(n)}, P_{6}=\left(x_{0}, y_{0}\right)$ is chosen so that

$$
0<x_{0}-\beta<\frac{1}{n}
$$

the distance between $P_{6}$ and $P_{7}$ is

$$
d\left[P_{6}, P_{7}\right]=\frac{\delta_{n}}{2}
$$

and if $P_{1}=f(-\alpha)+d$ where $0<d<\frac{1}{n}$. A similar condition is imposed on $P_{6}^{\prime}$ and $P_{7}^{\prime}$.

From the construction described in the proof of 1), it follows that

$$
\bigcap_{n} \mathfrak{A}\left(\varepsilon_{n}\right)=A B C D \text {. }
$$

Proof of 3 ): If $\varepsilon$ is sufficiently small, then $\frac{d x}{d t}$ is positive and bounded away from zero on the set

$$
U=\{(x, y) / y-f(x) \geq \delta\} .
$$

Hence, if $\left(x_{0}, y_{0}\right) \in U$, there is a number $\bar{t}_{0}>t_{0}$ such that $\left(x\left(\bar{t}_{0}\right), y\left(\bar{t}_{0}\right)\right)$ is on the curve

$$
y-f(x)=\delta
$$




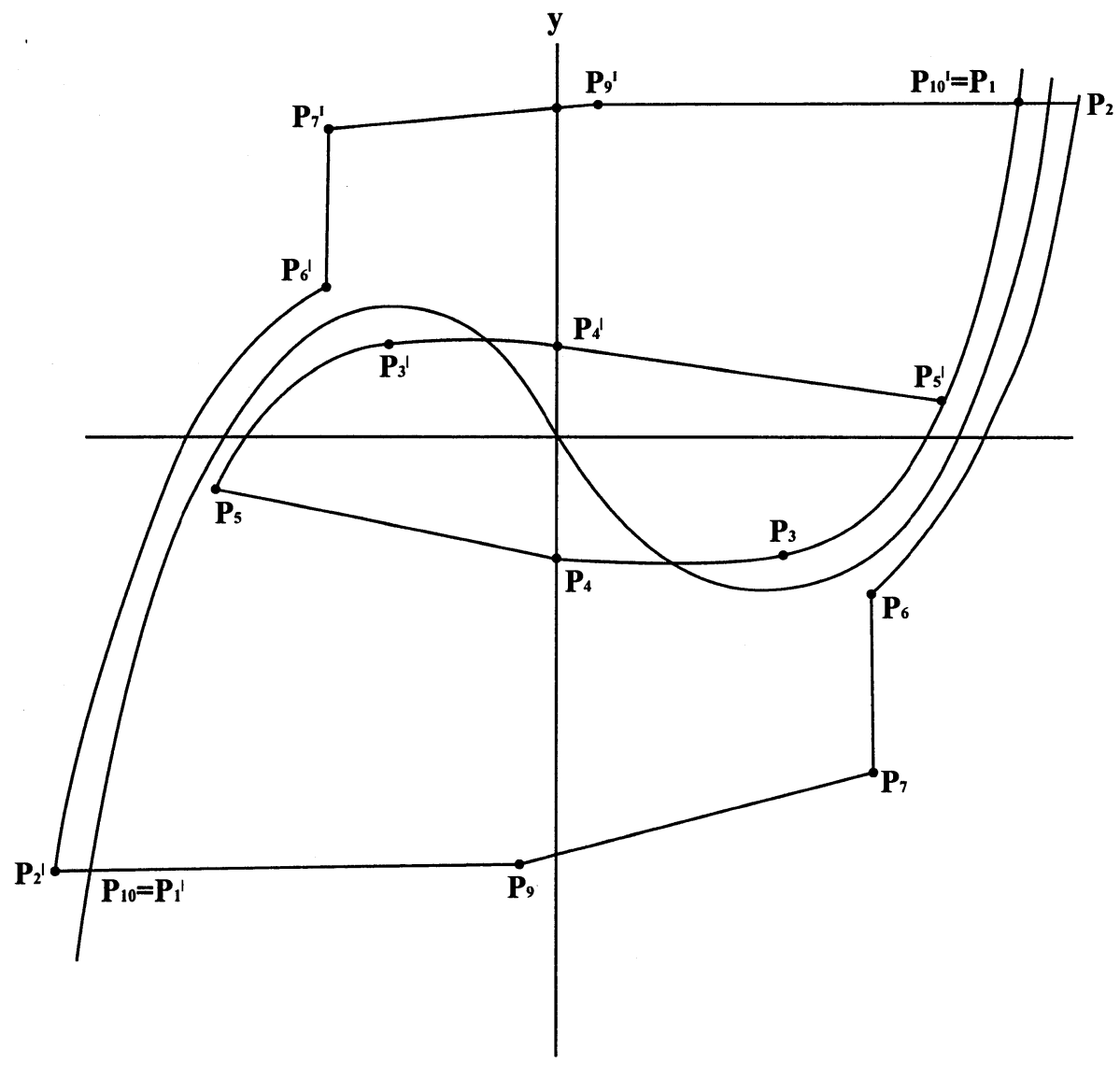

FiguRE 4

and if $t>\bar{t}_{0}$, then

$$
(x(t), y(t)) \in\{(x, y) / y-f(x)<\delta\} .
$$

Suppose $x\left(\bar{t}_{0}\right)>0$. Then for $t>\bar{t}_{0}$, we have $\frac{d y}{d t}(t)$ negative and bounded away from zero. Hence either

$$
\left(x\left(\bar{t}_{0}\right), y\left(\bar{t}_{0}\right)\right) \in \mathfrak{A}(\varepsilon)
$$

or there exists $\bar{t}_{1}<\bar{t}_{0}$ such that for $t>\bar{t}_{1}$

$$
(x(t), y(t)) \in \mathfrak{A}(\varepsilon) .
$$

Similar arguments hold for other cases.

Proof of 4): The neighborhood $N_{m}$ is the rhomboid-like figure in Figure 5 . The measure of $N_{m}$ can be made as small as desired by choosing $\delta$ small enough. If $\varepsilon>0$ is sufficiently small and the height of $N_{m}$ is sufficiently small, the orbits which intersect the boundary of $N_{m}$ cross the boundary going outward (as sketched).

(By the standard application of the Brouwer Fixed Point Theorem, it follows that there is a periodic orbit in $N_{m}$.)

To complete the proof, we need only show that if an orbit passes through a point

$$
\left(x_{0}, y_{0}\right) \in \operatorname{Int}\left(B_{i}\right)-N_{m},
$$




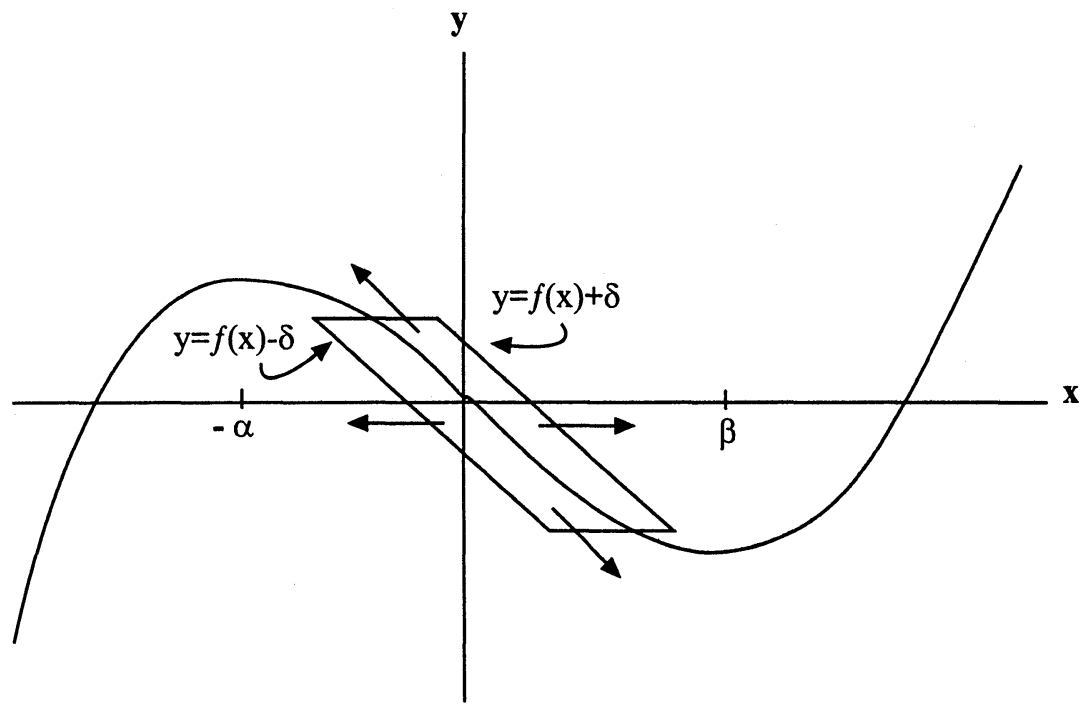

FiguRE 5

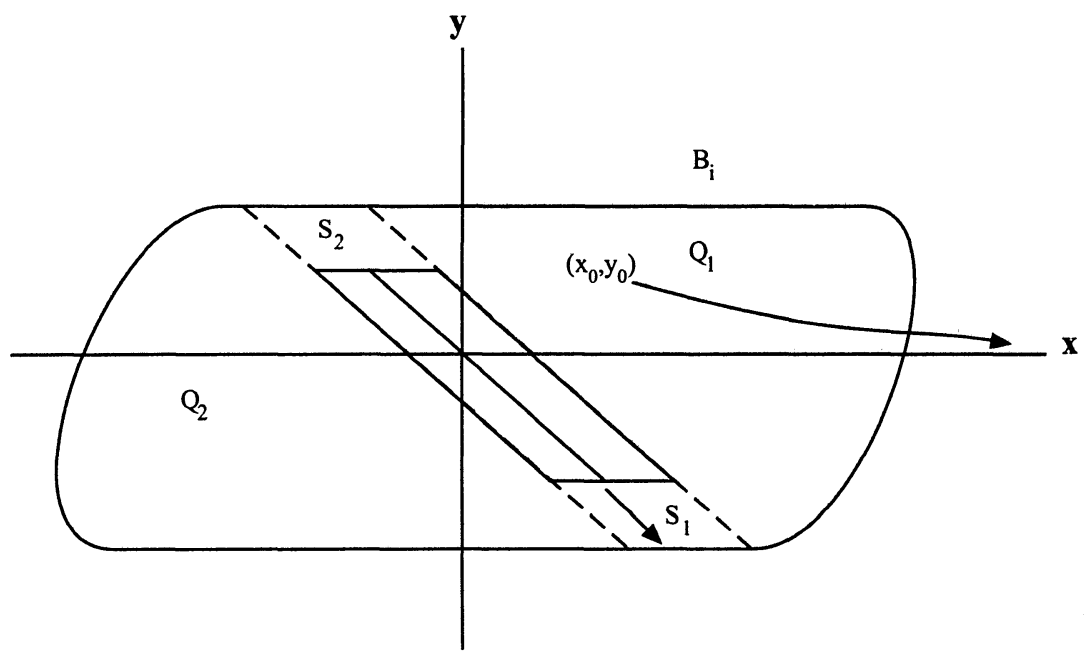

Figure 6

then the orbit enters $\mathfrak{A}\left(\varepsilon_{0}\right)$. Let

$$
Q_{1}=\left[\operatorname{Int}\left(B_{i}\right)\right] \cap\{(x, y) / y>f(x)+\delta\}
$$

(see Figure 6) and suppose $\left(x_{0}, y_{0}\right) \in Q_{1}$. If $\varepsilon$ is sufficiently small, then since $|d y / d t|$ is bounded in $\operatorname{Int}\left(B_{i}\right)$, the orbit will intersect $B_{i}$ (as sketched) before it can intersect the curve $y=f(x)+\delta$. Similarly, if

$$
Q_{2}=\left[\operatorname{Int}\left(B_{i}\right)\right] \cap\{(x, y) / y<f(x)=\delta\}
$$

and $\left(x_{0}, y_{0}\right) \in Q_{2}$, then if $\varepsilon$ is sufficiently small, the orbit will intersect $B_{i}$ before it can intersect the curve $y=f(x)-\delta$. Let

$$
S_{1}=\left[\operatorname{Int}\left(B_{i}\right)\right] \cap\{(x, y) / x>0, f(x)-\delta \leq y \leq f(x)+\delta\} .
$$


If $\left(x_{0}, y_{0}\right) \in S_{1}$ and the orbit later enters $Q_{1}$ or $Q_{2}$, the statements above apply. If the orbit remains in $S_{1}$, then since the $x$-coordinates of points in $S_{1}$ are positive and bounded away from zero, then the orbit intersects $B_{i}$ as sketched in Figure 6 . A similar argument can be used if

$$
\left(x_{0}, y_{0}\right) \in S_{2}=\left[\operatorname{Int}\left(B_{i}\right)\right] \cap\{(x, y) / x<0, f(x)-\delta \leq y \leq f(x)+\delta\} .
$$

Proof of 5): Now 5) follows at once from 3) and 4).

\section{References}

1. E. Aboufadel, Thesis, Rutgers University, 1992; A priori bounds for solutions of two-dimensional, singularly-perturbed, non-autonomous, ordinary differential equations (submitted for publication).

2. R.H. Adrian and L.D. Peachey, Reconstruction of the action potential of frog sartorius muscle, J. Physiol. 235 (1973), 103-131.

3. G.W. Beeler and H. Reuter, Reconstruction of the action potential of ventricular myocardial fibres, J. Physiol. 268 (1977), 177-210.

4. M.L. Cartwright, On non-linear differential equations of the second order III, Proc. Cambridge Phil. Soc. 45 (1949), 495-501.

5. M.L. Cartwright and J.E. Littlewood, On non-linear differential equations of the second order I, J. London Math. Soc. 20 (1945), 180-189.

6. - On non-linear differential equations of the second order II, Annals of Math. 48 (1947), $472-494$.

7. L. Cesari, Asymptotic Behavior and Stability Problems in Ordinary Differential Equations, Springer-Verlag, Berlin, 1959.

8. E. A. Coddington and N. Levinson, Theory of Ordinary Differential Equations, McGraw-Hill, New York, 1955.

9. J. Cronin, Fixed Points and Topological Degree in Nonlinear Analysis, American Mathematical Society, Providence, Rhode Island, 1964.

10. __ Mathematical Aspects of Hodgkin-Huxley Neural Theory, Cambridge University Press, Cambridge, 1987.

11. _ Differential Equations: Introduction and Qualitative Theory, Second edition, Marcel Dekker, New York, 1994.

12. D. DiFrancesco and D. Noble, A model of cardiac electrical activity incorporating ionic pumps and concentration changes, Phil. Trans. R. Soc. Lond. B. 240 (1985), 83-96.

13. D.A. Flanders and J.J. Stokes, The limit case of relaxation oscillations, in Studies in Nonlinear Vibration Theory, (Ed. R. Courant), Institute for Math. and Mech., NYU, 1946, pp. 51-64.

14. B. Frankenhaeuser and A. F. Huxley, The action potential in the myelinated nerve fibre of Xenopus laevis as computed on the basis of voltage clamp data, J. Physiol. 171 (1964), 302-315.

15. J. K. Hale, Oscillations in Nonlinear Systems, McGraw-Hill, New York, 1963.

16. __ Ordinary Differential Equations, Robert E. Krieger Publishing Co. Inc., Malabar, Florida, 1980.

17. A. L. Hodgkin and A. F. Huxley, A quantitative description of membrane current and its application to conduction and excitation in nerve, J. Physiol. 117 (1952), 500-544.

18. J. LaSalle, Relaxation oscillations, Quarterly Appl. Math. 7 (1949), 1-19.

19. E. Leimanis and N. Minorsky, Dynamics and Nonlinear Mechanics, John Wiley \& Sons, Inc., New York, 1958.

20. N. Levinson, Perburbations of discontinuous solutions of non-linear systems of differential equations, Acta Math. 82 (1951), 71-106.

21. J.E. Littlewood, On non-linear differential equations of the second order III, Acta Math. 97 (1957), 267-308.

22. R.E. McAllister, D. Noble, and R.W. Tsien, Reconstruction of the electrical activity of cardiac Purkinje fibres, J. Physiol. 251 (1975), 1-58.

23. I.G. Malkin, Some Problems in the Theory of Nonlinear Oscillations, State Publishing House of Technical and Theoretical Literature, Moscow, 1956. (Translated by The AEC, 1959.)

24. N. Minorsky, Introduction to Non-linear Mechanics, J.W. Edwards, Ann Arbor, Michigan, 1947. 25 . Nonlinear Oscillations, D. Van Nostrand Company, Inc., Princeton, New Jersey, 1962. 
26. E.F. Mishchenko and N.Kh. Rozov, Differential Equations with Small Parameters and Relaxation Oscillations, (translated from Russian by F.M.C. Goodspeed), Plenum Press, New York, 1980.

27. D. Noble, A modification of the Hodgkin-Huxley equations applicable to Purkinje fibre action and pace-maker potentials, J. Physiol. 160 (1962), 317-352.

28. __ OXSOFT Heart Program Manual, OXSOFT Ltd., Oxford, England, 1990.

29. D. Noble and S. Noble, A model of sino-atrial node electrical activity based on a modification of the DiFrancesco-Noble (1984) equations, Proc. R. Soc. B222 (1984), 295-304.

30. Henri Poincaré, Les méthodes nouvelles de la mécanique céleste, 3 volumes, Paris, GauthiersVillars, 1892-99. Reprinted by Dover Publications, New York, 1958.

31. M. Roseau, Vibrations non linéaires et théoriè de la stabilité, Springer-Verlag, Berlin, Heidelberg, New York, 1966.

32. _ Vibrations in Mechanical Systems (translated from French by H.L.S. Orde), SpringerVerlag, Berlin, Heidelberg, New York, 1987.

33. J.J. Stoker, Nonlinear vibrations in mechanical and electrical systems, Interscience Publishers Inc., New York, 1950.

34. R.D. Traub and R. Miles, Neuronal Networks of the Hippocampus, Cambridge University Press, Cambridge, 1991.

35. B. van der Pol and J. van der Mark, The heartbeat considered as a relaxation oscillation and an electrical model of the heart, Phil. Mag. 6 (1928), 763-775.

36. K. Yanagihara, A. Noma, and H. Irisawa, Reconstruction of sinoatrial node pacemaker potential based on the voltage-clamp experiments, Jpn. J. Physiol. 30 (1980), 841-857.

Department of Mathematics, Rutgers University, New Brunswick, NJ 08903, U.S.A. 